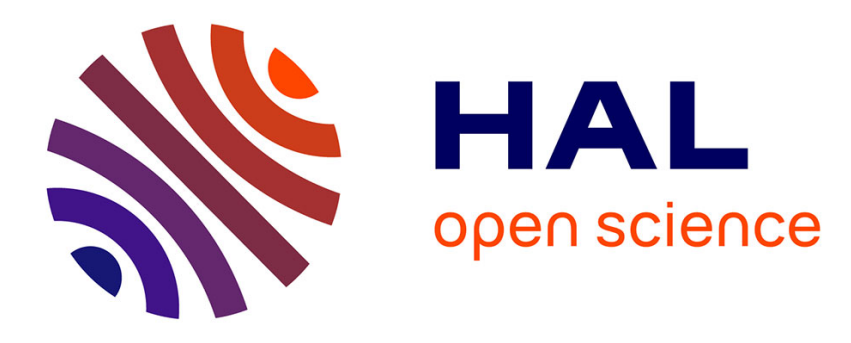

\title{
Rigid multibody system dynamics with uncertain rigid bodies
}

\author{
Anas Batou, Christian Soize
}

\section{To cite this version:}

Anas Batou, Christian Soize. Rigid multibody system dynamics with uncertain rigid bodies. Multibody System Dynamics, 2012, 27 (3), pp.285-319. 10.1007/s11044-011-9279-2 . hal-00743648

\section{HAL Id: hal-00743648 \\ https://hal.science/hal-00743648}

Submitted on 19 Oct 2012

HAL is a multi-disciplinary open access archive for the deposit and dissemination of scientific research documents, whether they are published or not. The documents may come from teaching and research institutions in France or abroad, or from public or private research centers.
L'archive ouverte pluridisciplinaire HAL, est destinée au dépôt et à la diffusion de documents scientifiques de niveau recherche, publiés ou non, émanant des établissements d'enseignement et de recherche français ou étrangers, des laboratoires publics ou privés. 


\title{
Rigid multibody system dynamics with uncertain rigid bodies
}

\author{
A. Batou · C. Soize
}

Received: date / Accepted: date

\begin{abstract}
This paper is devoted to the construction of a probabilistic model of uncertain rigid bodies for multibody system dynamics. We first construct a stochastic model of an uncertain rigid body by replacing the mass, the center of mass and the tensor of inertia by random variables. The prior probability distributions of the stochastic model are constructed using the maximum entropy principle under the constraints defined by the available information. The generators of independent realizations corresponding to the prior probability distribution of these random quantities are further developed. Then, several uncertain rigid bodies can be linked to each other in order to calculate the random response of a multibody dynamical system. An application is proposed to illustrate the theoretical development.
\end{abstract}

Keywords Uncertain rigid body $\cdot$ Random mass $\cdot$ Random center of mass $\cdot$ Random tensor of inertia.

\section{Nomenclature}

A. Batou, C. Soize

Université Paris-Est, Laboratoire Modélisation et Simulation Multi Echelle, MSME UMR 8208 CNRS,

5 bd Descartes, 77454 Marne-la-Vallee, France

Tel: 33160957661

Fax: 33160957799

E-mail: anas.batou@univ-paris-est.fr , christian.soize@univ-paris-est.fr 
h Vector of the parameters describing the domain $\mathcal{D}_{i}$.

$\mathbf{k} \quad$ Vector of the Coriolis forces $(\mathrm{Nm})$.

$m_{i} \quad$ Mass of $\mathrm{RB}_{i}(\mathrm{~kg})$.

$\underline{m}_{i} \quad$ Nominal value of the mass of $\mathrm{RB}_{i}(\mathrm{~kg})$.

$n_{b} \quad$ Number of rigid bodies.

$n_{c} \quad$ Number of holonomic constraints.

$\mathbf{q} \quad$ Vector of the applied forces and torques $(N$ and $N m)$.

$\mathbf{r}_{i} \quad$ Position vector of $\mathcal{G}_{i}(m)$.

$\mathbf{r}_{0, i} \quad$ Initial position of $\mathcal{G}_{i}(m)$.

$\underline{\mathbf{r}}_{0, i} \quad$ Initial position of $\underline{\mathcal{G}}_{i}(m)$.

$\mathbf{r}_{b, i} \quad$ Position vector of the barycenter of the domain $\mathcal{D}_{i}(m)$.

$\mathbf{s}_{i} \quad$ Rotation vector of $\mathrm{RB}_{i}(\mathrm{rad})$.

$\mathbf{s}_{0, i} \quad$ Initial angular position of $\mathrm{RB}_{i}(\mathrm{rad})$.

$\mathbf{u} \quad$ Vector of the position and angle of the centers of mass ( $m$ and $\mathrm{rad}$ ).

$\mathbf{v}_{0, i} \quad$ Initial velocity of $\mathcal{G}_{i}(\mathrm{~m} / \mathrm{s})$.

$\mathbf{x} \quad$ Position vector in the inertial frame $(m)$.

$\mathbf{x}^{\prime} \quad$ Position vector in the local frame $(m)$.

$C_{0} \quad$ Normalisation constant relative to $\mathbf{R}_{0, i}$.

$C_{G_{i}} \quad$ Normalisation constant relative to $\left[\mathbf{G}_{i}\right]$.

$C_{G_{0, i}} \quad$ Normalisation constant relative to $\left[\mathbf{G}_{0, i}\right]$.

$\mathcal{D}_{i} \quad$ Admissible domain of $\mathbf{R}_{0, i}$.

$\mathcal{G}_{i} \quad$ Center of mass of $\mathrm{RB}_{i}$.

$\underline{\mathcal{G}}_{i} \quad$ Center of mass of the nominal model of $\mathrm{RB}_{i}$.

$\overline{\mathcal{G}}_{i} \quad$ Random center of mass of the probabilistic model of $\mathrm{RB}_{i}$.

$\left[\mathbf{G}_{i}\right] \quad$ Normalized positive definite bounded $(3 \times 3)$ random matrix.

$\left[G_{i}^{\max }\right] \quad$ Upper Bound for random matrix $\left[\mathbf{G}_{i}\right]$.

$\left[\mathbf{G}_{0, i}\right] \quad$ Normalized positive definite $(3 \times 3)$ random matrix.

$\left[H_{i}\right] \quad$ Second order moment of inertia of $\mathrm{RB}_{i}\left(\mathrm{~kg} \mathrm{~m}^{2}\right)$.

$\left[J_{i}\right] \quad$ Tensor of inertia of $\mathrm{RB}_{i}\left(\mathrm{~kg} \mathrm{~m}^{2}\right)$.

$\left[\widetilde{J}_{i}\right] \quad$ Tensor of inertia of $\mathrm{RB}_{i}$ with unit mass $\left(\mathrm{m}^{2}\right)$.

$\left[\underline{J}_{i}\right] \quad$ Nominal value of the tensor of inertia of $\mathrm{RB}_{i}\left(\mathrm{~kg} \mathrm{~m}^{2}\right)$.

$\left[\mathbf{J}_{i}\right]$ Random tensor of inertia of the probabilistic model of $\mathrm{RB}_{i}\left(\mathrm{~kg} \mathrm{~m}^{2}\right)$.

$\left[\mathbf{J}_{i}^{\max }\right] \quad$ Random upper Bound for random matrix $\left[\mathbf{J}_{i}\right]\left(\mathrm{kg} \mathrm{m}^{2}\right)$.

$\mathbf{K}$ Random vector of the Coriolis forces $(\mathrm{Nm})$.

$\left[\underline{L}_{Z_{i}}\right] \quad$ Upper triangular matrix relative to the Cholesky factorisation of $\left[\underline{Z}_{i}\right](\mathrm{m})$.

$[M] \quad$ Mass matrix $(\mathrm{kg})$.

[M] Random mass matrix $(\mathrm{kg})$.

$M_{i} \quad$ Random mass of the probabilistic model of $\mathrm{RB}_{i}(\mathrm{~kg})$.

$\mathbf{R}_{0, i} \quad$ Random initial position of $\mathcal{G}_{i}(m)$.

$\mathrm{RB}_{i} \quad$ Rigid body $i$.

U Random vector of the position and angle of the centers of mass ( $m$ and $\mathrm{rad}$ ).

$\left[Z_{i}\right] \quad$ Second order moment of inertia of $\mathrm{RB}_{i}$ with unit mass $\left(m^{2}\right)$.

$\left[\underline{Z}_{i}\right] \quad$ Nominal value of $\left[Z_{i}\right]$.

$\left[\mathbf{Z}_{i}\right] \quad$ Random second order moment of inertia of $\mathrm{RB}_{i}$ with unit mass $\left(m^{2}\right)$.

$\left[Z_{i}^{\max }\right] \quad$ Upper Bound for random matrix $\left[\mathbf{Z}_{i}\right]\left(\mathrm{m}^{2}\right)$.

$\delta_{M_{i}} \quad$ Coeficient of variation for $M_{i}$.

$\lambda \quad$ Real-valued lagrange multiplier relative to $\left[\mathbf{G}_{0, i}\right]$.

$\boldsymbol{\lambda} \quad$ Vector of the Lagrange multipliers of the constraints $(N)$.

$\lambda_{l}, \lambda_{u} \quad$ Real-valued lagrange multipliers relative to $\left[\mathbf{G}_{i}\right]$.

$[\mu] \quad$ Matrix-valued lagrange multiplier relative to $\left[\mathbf{G}_{i}\right]$.

$\left[\mu_{0}\right]$ Matrix-valued lagrange multiplier relative to $\left[\mathbf{G}_{0, i}\right]$.

$\boldsymbol{\lambda}_{r} \quad$ Lagrange multipliers relative to $\mathbf{R}_{0, i}\left(m^{-1}\right)$.

$\rho \quad$ Mass density $\left(\mathrm{kg} / \mathrm{m}^{3}\right)$.

$\varphi \quad$ Constraint function.

$\boldsymbol{\omega}_{i} \quad$ Angular velocity of $\mathrm{RB}_{i}(\mathrm{rad} / \mathrm{s})$.

$\boldsymbol{\omega}_{0, i} \quad$ Initial angular velocity of $\mathrm{RB}_{i}(\mathrm{rad} / \mathrm{s})$.

$\Gamma \quad$ Gamma function.

$\Omega_{i} \quad$ Domain of $\mathrm{RB}_{i}$. 


\section{Introduction}

The study of rigid multibody dynamical systems has interested numerous researchers during the last three decades. The efficient methods developed during this period are now implemented in commercial softwares and are commonly used in many application fields such as automotive vehicles, railway vehicles, launch vehicles and so on.

Probability theory has been intensively used over the last two decades to model uncertainties in structural dynamics and vibration (see for instance [25]). However, there are very few published results concerning the modeling of uncertainties in multibody dynamics, in particular to take into account an uncertain spatial mass distribution inside a rigid body. These uncertainties in the spatial mass distribution induce uncertainties in the mass, the position of the center of mass and the tensor of inertia. In this paper, an uncertain rigid body is a rigid body for which its mass, its center of mass and its tensor of inertia are uncertain. A complete probabilistic model of uncertainties is proposed for the first time in order to take into account uncertain rigid bodies in multibody dynamical systems.

In some cases, the mass distribution inside a rigid body is not perfectly known and must be considered as random (for example, the distribution of passengers inside a vehicle) and therefore, this unknown mass distribution inside the rigid body induces uncertainties in the model of this rigid body. In this paper, we propose a new probabilistic modeling for uncertain rigid bodies in the context of the multibody dynamics. Concerning the modeling of uncertainties in multibody dynamical system, very few previous studies have been carried out. They aimed at taking into account uncertainties (1) for parameters such as stiffness of a suspension [13], friction coefficient [24], aerodynamic coefficients [3]), (2) for the input loads or imposed displacements such as, for instance, the profile of a road (see [18] and $[20,21,3]$ ). Note that these types of parameters describe the joints linking each rigid body to the others and the external sources, but not rigid bodies themselves. In the field of uncertain rigid bodies, a first work has been proposed in $[15,16]$, in which the authors take into account uncertain rigid bodies for rotor dynamical systems using the nonparametric probabilistic approach $[28,29]$ consisting in replacing the mass and gyroscopic matrices by random matrices. It should be noted that this paper is focused on the construction of the stochastic modeling of uncertainties for the rigid bodies. It is then assumed that there are no uncertainties for the model parameters of the mechanical links between the rigid bodies. Nevertheless, if such uncertainties existed in the mechanical links, the construction of their stochastic models could be carried out using the usual parametric approach (see for instance [24]) and can be added without difficulties to the present theory. Similarly, it is straightforward to add uncertainties on external sources. In this paper, a general and complete stochastic model is constructed for an uncertain rigid body. The mass, the center of mass and the tensor of inertia which describe the rigid body are modeled by random variables. The prior probability distributions of the random variables are constructed using the maximum entropy principle [11] from Information Theory [27]. The generator of independent realizations corresponding to the prior probability distributions of these random quantities are developed and presented. Then, several uncertain rigid bodies can be linked to each other in order to calculate the random response of an uncertain multibody dynamical system. The stochastic multibody dynamical equations are solved using the Monte Carlo simulation method. The statistical moments and the confidence regions of the random responses are estimated using mathematical statistics. 
Section 2 is devoted to the construction of the nominal model for the rigid multibody dynamical system by using the classical method. In Section 3, we propose a general probability model for an unconstrained uncertain rigid body and then, the uncertain rigid multibody dynamical system is obtained by joining this unconstrained uncertain rigid body to the other rigid bodies. The last section is devoted to an application which illustrates the proposed theory.

\section{Nominal model for the rigid multibody dynamical system}

In this paper, the usual model of a rigid multibody dynamical system for which all the mechanical properties are known will be called the nominal model. This section is devoted to the construction of the nominal model for a rigid multibody dynamical system. This nominal model is constructed as in $[10,22,23]$ and is summarized below.

2.1 Dynamical equations for a rigid body of the multibody system

Let $\mathrm{RB}_{i}$ be the rigid body occupying a bounded domain $\Omega_{i}$ with a given geometry. Let $\xi$ be the generic point of the three dimensional space. Let $\mathbf{x}=\left(x_{1}, x_{2}, x_{3}\right)$ be the position vector of point $\xi$ defined in a fixed inertial frame $\left(O, x_{0,1}, x_{0,2}, x_{0,3}\right)$, such that $\mathbf{x}=\overrightarrow{O \xi}$. Let $d \mathbf{x}$ be the Lebesgue measure (elementary volume). The rigid body class is then defined by three quantities.

(1) The first one is the mass $m_{i}$ of $\mathrm{RB}_{i}$ which is such that

$$
m_{i}=\int_{\Omega_{i}} \rho(\mathbf{x}) d \mathbf{x}
$$

where $\rho(\mathbf{x})$ is the mass density. If body $\mathrm{RB}_{i}$ is made up of $n$ discrete masses $\mu_{1}, \ldots, \mu_{n}$ located at $\mathbf{x}_{1}, \ldots, \mathbf{x}_{n}$ in $\Omega_{i}$, then $\rho(\mathbf{x}) d \mathbf{x}=\sum_{j=1}^{n} \mu_{j} \delta_{\mathbf{x}_{j}}(\mathbf{x})$ in which $\delta_{\mathbf{x}_{j}}(\mathbf{x})$ is such that for all real-valued continuous functions $g(\mathbf{x})$, we have $\int_{\Omega_{i}} g(\mathbf{x}) \delta_{\mathbf{x}_{j}}(\mathbf{x})=g\left(\mathbf{x}_{j}\right)$.

(2) The second quantity is the position vector $\mathbf{r}_{i}$ of the center of mass $\mathcal{G}_{i}$, defined in the fixed inertial frame, by

$$
\mathbf{r}_{i}=\frac{1}{m_{i}} \int_{\Omega_{i}} \mathbf{x} \rho(\mathbf{x}) d \mathbf{x}
$$

(3) Let $\left(\mathcal{G}_{i}, x_{i, 1}^{\prime}, x_{i, 2}^{\prime}, x_{i, 3}^{\prime}\right)$ be the local frame for which the origin is $\mathcal{G}_{i}$ and which is deduced from the fixed frame $\left(O, x_{0,1}, x_{0,2}, x_{0,3}\right)$ by the translation $\overrightarrow{O_{\mathcal{G}}}$ and a rotation defined by the three Euler angles $\alpha_{i}, \beta_{i}$ and $\gamma_{i}$. The third quantity is the positive-definite matrix $\left[J_{i}\right]$ of the tensor of inertia in the local frame such that

$$
\left[J_{i}\right] \mathbf{u}=-\int_{\Omega_{i}} \mathbf{x}^{\prime} \times \mathbf{x}^{\prime} \times \mathbf{u} \rho\left(\mathbf{x}^{\prime}\right) d \mathbf{x}^{\prime} \quad, \quad \forall \mathbf{u} \in \mathbb{R}^{3}
$$

in which the vector $\mathbf{x}^{\prime}=\left(x_{1}^{\prime}, x_{2}^{\prime}, x_{3}^{\prime}\right)$ of the components of vector $\overrightarrow{\mathcal{G}_{i} \xi}$ are given in $\left(\mathcal{G}_{i}, x_{i, 1}^{\prime}, x_{i, 2}^{\prime}, x_{i, 3}^{\prime}\right)$. In the above equation, $\mathbf{u} \times \mathbf{v}$ denotes the cross product between 
the vectors $\mathbf{u}$ and $\mathbf{v}$. Applying Newton and Euler equations to rigid body $\mathrm{RB}_{i}$, yields, for all $t$ in the time interval $[0, T]$, the following differential equations

$$
\begin{array}{r}
m_{i} \ddot{\mathbf{r}}_{i}=\mathbf{f}_{i}^{e}+\mathbf{f}_{i}^{r}, \\
{\left[J_{i}\right] \dot{\boldsymbol{\omega}}_{i}+\mathbf{k}_{i}=\mathbf{l}_{i}^{e}+\mathbf{l}_{i}^{r},}
\end{array}
$$

with the initial conditions

$$
\begin{gathered}
\mathbf{r}_{i}(0)=\mathbf{r}_{0, i} \quad, \quad \dot{\mathbf{r}}_{i}(0)=\mathbf{v}_{0, i} \quad, \\
\mathbf{s}_{i}(0)=\mathbf{s}_{0, i} \quad, \quad \boldsymbol{\omega}_{i}(0)=\boldsymbol{\omega}_{0, i} \quad .
\end{gathered}
$$

It should be noted that Eq. (4) is written in the fixed inertial frame $\left(O, x_{0,1}, x_{0,2}\right.$, $\left.x_{0,3}\right)$ while Eq. (5) is written in the local frame $\left(\mathcal{G}_{i}, x_{i, 1}^{\prime}, x_{i, 2}^{\prime}, x_{i, 3}^{\prime}\right)$. In these equations, $\mathbf{k}_{i}\left(\boldsymbol{\omega}_{i},\left[J_{i}\right]\right)=\boldsymbol{\omega}_{i} \times\left\{\left[J_{i}\right] \boldsymbol{\omega}_{i}\right\}$ is the vector of the Coriolis forces, $\mathbf{l}_{i}^{e}$ and $\mathbf{f}_{i}^{e}$ are the vectors of the applied torque reduced at $\mathcal{G}_{i}$ and the applied force, where $\mathbf{l}_{i}^{r}$ and $\mathbf{f}_{i}^{r}$ are the constraint torque reduced at $\mathcal{G}_{i}$ and the constraint force, where $\boldsymbol{\omega}_{i}=\dot{\mathbf{s}}_{i}$ is the angular velocity vector in the local frame with $\mathbf{s}_{i}=\left(\alpha_{i}, \beta_{i}, \gamma_{i}\right)$ the rotation vector. The vector $\mathbf{r}_{0, i}$ defines the initial position of the center of mass while $\mathbf{v}_{0, i}$ defines its initial velocity. The vector $\mathbf{s}_{0, i}$ defines the initial angular position and $\boldsymbol{\omega}_{0, i}$ defines the initial angular velocity.

\subsection{Matrix model for the rigid multibody dynamical system}

The rigid multibody dynamical system is made up of $n_{b}$ rigid bodies and ideal joints including rigid joints, joints with given motion (rheonomic constraints) and vanishing joints (free motion). The interactions between the rigid bodies are realized by these ideal joints but also by springs, dampers or actuators which produce forces between the bodies. In this paper, only $n_{c}$ holonomic constraints are considered. Let $\mathbf{u}$ be the vector in $\mathbb{R}^{6 n_{b}}$ such that $\mathbf{u}=\left(\mathbf{r}_{1}, \ldots, \mathbf{r}_{n_{b}}, \mathbf{s}_{1}, \ldots, \mathbf{s}_{n_{b}}\right)$. The $n_{c}$ constraints are given by $n_{c}$ implicit equations which are globally written as

$$
\varphi(\mathbf{u}, t)=0
$$

The function $\{\mathbf{u}(t), \in[0, T]\}$ is the solution of the following differential equation

$$
[M] \ddot{\mathbf{u}}(t)+\mathbf{k}(\dot{\mathbf{u}}(t))=\mathbf{q}(\mathbf{u}(t), \dot{\mathbf{u}}(t), t)-\left[\boldsymbol{\varphi}_{\mathbf{u}}(\mathbf{u}(t), t)\right]^{T} \boldsymbol{\lambda}(t)
$$

with the initial conditions

$$
\mathbf{u}(0)=\mathbf{u}_{0} \quad, \quad \dot{\mathbf{u}}(0)=\mathbf{v}_{0} \quad,
$$

in which $\dot{\mathbf{u}}=d \mathbf{u} / d t, \ddot{\mathbf{u}}=d^{2} \mathbf{u} / d t^{2}$, where $\mathbf{u}_{0}=\left(\mathbf{r}_{0,1}, \ldots, \mathbf{r}_{0, n_{b}}, \mathbf{s}_{0,1}, \ldots, \mathbf{s}_{0, n_{b}}\right)$, in which $\mathbf{v}_{0}=\left(\mathbf{v}_{0,1}, \ldots, \mathbf{v}_{0, n_{b}}, \boldsymbol{\omega}_{0, i}, \ldots, \boldsymbol{\omega}_{0, n_{b}}\right)$ and where $\mathbf{k}(\dot{\mathbf{u}})=\left(\mathbf{0}, \ldots, \mathbf{0}, \mathbf{k}_{1}\left(\dot{\mathbf{s}}_{1},\left[J_{1}\right]\right), \ldots\right.$, $\left.\mathbf{k}_{n_{b}}\left(\dot{\mathbf{s}}_{n_{b}},\left[J_{n_{b}}\right]\right)\right)$ is the $\mathbb{R}^{6 n_{b}}$-vector of the Coriolis forces. The $\mathbb{R}^{6 n_{b}}$-vector $\mathbf{q}(\mathbf{u}, \dot{\mathbf{u}}, t)$ is such that $\mathbf{q}(\mathbf{u}, \dot{\mathbf{u}}, t)=\left(\mathbf{f}_{1}^{e}(\mathbf{u}, \dot{\mathbf{u}}, t), \ldots, \mathbf{f}_{n_{b}}^{e}(\mathbf{u}, \dot{\mathbf{u}}, t), \mathbf{l}_{1}^{e}(\mathbf{u}, \dot{\mathbf{u}}, t), \ldots, \mathbf{l}_{n_{b}}^{e}(\mathbf{u}, \dot{\mathbf{u}}, t)\right)$ constituted of the applied forces and torques induced by springs, dampers and actuators. The vector $\left[\boldsymbol{\varphi}_{\mathbf{u}}(\mathbf{u}(t), t)\right]^{T} \boldsymbol{\lambda}(t)$ is relative to the constraint forces where $\boldsymbol{\lambda}(t)$ is the vector of the Lagrange multipliers and $\left[\boldsymbol{\varphi}_{\mathbf{u}}(\mathbf{u}(t), t)\right]$ is the Jacobian matrix of $\boldsymbol{\varphi}(\mathbf{u}(t), t)$ with 
respect to $\mathbf{u}(t)$ such that $\left[\boldsymbol{\varphi}_{\mathbf{u}}(\mathbf{u}(t), t)\right]_{i j}=\partial \varphi_{i}(\mathbf{u}(t), t) / \partial u_{j}(t)$. The $\left(6 n_{b} \times 6 n_{b}\right)$ mass matrix $[M]$ is defined by

$$
[M]=\left[\begin{array}{cc}
{\left[M^{r}\right]} & 0 \\
0 & {\left[M^{s}\right]}
\end{array}\right],
$$

where the $\left(3 n_{b} \times 3 n_{b}\right)$ matrices $\left[M^{r}\right]$ and $\left[M^{s}\right]$ are defined by

$$
\left[M^{r}\right]=\left[\begin{array}{ccc}
m_{1}\left[I_{3}\right] & \cdots & 0 \\
\vdots & \ddots & \vdots \\
0 & \cdots & m_{n_{b}}\left[I_{3}\right]
\end{array}\right] \quad, \quad\left[M^{s}\right]=\left[\begin{array}{ccc}
{\left[J_{1}\right]} & \cdots & 0 \\
\vdots & \ddots & \vdots \\
0 & \cdots & {\left[J_{n_{b}}\right]}
\end{array}\right],
$$

in which $\left[I_{3}\right]$ is the $(3 \times 3)$ identity matrix. To solve Eq. (9) with Eq. (8), a twice differentiation of the constraint Eq. (8) with respect to $t$ is performed and yields

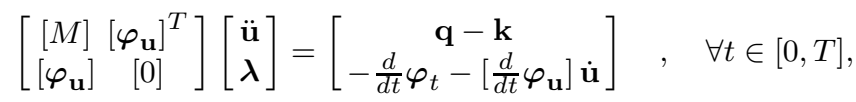

with the initial conditions

$$
\mathbf{u}(0)=\mathbf{u}_{0} \quad, \quad \dot{\mathbf{u}}(0)=\mathbf{v}_{0} \quad,
$$

in which $\varphi_{t}=\partial \varphi / \partial t$. Equation (13) can be solved using an adapted integration algorithm (see for instance [2]).

\section{Stochastic model for a multibody dynamical system with uncertain rigid bodies}

Firstly, a stochastic model for an uncertain rigid body of the multibody dynamical system is proposed and secondly, this stochastic model is introduced in the nominal model to obtain the stochastic model for the multibody dynamical system with uncertain rigid bodies.

3.1 Stochastic model for an uncertain rigid body of the multibody dynamical system

The mass, the initial position vector of the center of mass $\mathcal{G}_{i}$ and the matrix of the tensor of inertia of the nominal model of the uncertain rigid body $\mathrm{RB}_{i}$ are respectively denoted by $\underline{m}_{i}, \underline{\mathbf{r}}_{0, i}$ and $\left[\underline{J}_{i}\right]$. The probabilistic model of uncertainties for this rigid body is constructed by replacing these three parameters by the following three random variables: the random mass $M_{i}$, the random initial position vector $\mathbf{R}_{0, i}$ of its random center of mass $\mathcal{G}_{i}$ and the random matrix $\left[\mathbf{J}_{i}\right]$ of its random tensor of inertia with respect to the random local frame $\left(\mathcal{G}_{i}, \underline{x}_{i, 1}^{\prime}, \underline{x}_{i, 2}^{\prime}, \underline{x}_{i, 3}^{\prime}\right)$. The probability density functions (PDF) of these three random variables are constructed using the maximum entropy principle (see [27], [11]), that is to say, in maximizing the uncertainties in the model under the constraints defined by the available information. Below, the available informations, devoted to the existence of the second-order moment of the norm of the inverse of the random quantities, are introduced for that the linearized stochastic dynamical system admits a second-order solution (physical solution)[29]. We will assume that such constraints are sufficient to guaranty the existence of a second-order solution of the non-linear stochastic dynamical system. 
3.1.1 Construction of the PDF for the random mass

In this subsection, the PDF of the random mass $M_{i}$ is constructed using the maximum entropy principle under the constraints defined by the available information. Therefore, in a first step, this available information is deduced from physical considerations. Then, in a second step, the PDF of the random mass $M_{i}$ is obtained as the function maximizing the entropy (which is a functional of the PDF).

\section{(i) Available information}

Let $E\{$.$\} be the mathematical expectation. For the random mass M_{i}$, the available information is defined as follows. Since a mass is a positive quantity, $M_{i}$ is a random variable which must be with positive values. In addition, the nominal model would be the model which would be used if no uncertainties were taken into account. Consequently, the nominal model has to be considered as the mean model of the stochastic model that we are constructing. It should be noted that the mean response of the stochastic model is not equal to response of the nominal (mean) model. Presently, we only write that the mean values of the random parameters of the uncertain multibody dynamical system are equal to the nominal parameters. In this framework and by construction, the mean value of the random mass $M_{i}$ is then chosen as the value $\underline{m}_{i}$ of the nominal model. Finally, as it is proven in [29], the random mass must verify the inequality $E\left\{M_{i}^{-2}\right\}<+\infty$ in order that a second-order solution exists for the stochastic multibody dynamical system. It is also proven that this constraint can be replaced by the following one, $\left|E\left\{\log M_{i}\right\}\right|<+\infty$. In these conditions, the available information can be summarized as follows

$$
\begin{gathered}
M_{i}>0, \\
E\left\{M_{i}\right\}=\underline{m}_{i}, \\
E\left\{\log M_{i}\right\}=C_{M_{i}} \quad, \quad\left|C_{M}\right|<+\infty .
\end{gathered}
$$

(ii) Maximum entropy principle

The probability density function $\mu \mapsto p_{M_{i}}(\mu)$ of the random variable $M_{i}$ is constructed by maximizing the entropy defined by

$$
\mathcal{S}\left(p_{M_{i}}\right)=-\int_{\mathbb{R}} p_{M_{i}}(\mu) \log \left(p_{M_{i}}(\mu)\right) d \mu,
$$

under the constraints defined by Eq. (15). The solution of this optimization problem is the PDF of a gamma random variable defined on $] 0,+\infty[$. This PDF depends on two parameters which are $\underline{m}_{i}$ and $C_{M_{i}}$. Since the parameter $C_{M_{i}}$ has no physical meaning, it is eliminated in introducing the coefficient of variation $\delta_{M_{i}}$ of the random variable $M_{i}$ such that $\delta_{M_{i}}=\sigma_{M_{i}} / \underline{m}_{i}$ where $\sigma_{M_{i}}$ is the standard deviation of the random variable $M_{i}$. The following PDF is then obtained,

$$
p_{M_{i}}\left(\mu ; \delta_{M_{i}}\right)=\mathbb{1}_{] 0,+\infty[}(\mu) \frac{1}{\underline{m}_{i}}\left(\frac{1}{\delta_{M_{i}}^{2}}\right)^{\delta_{M_{i}}^{-2}} \frac{1}{\Gamma\left(\delta_{M_{i}}^{-2}\right)}\left(\frac{\mu}{\underline{m}_{i}}\right)^{\delta_{M_{i}}^{-2}-1} \exp \left(-\frac{\mu}{\delta_{M_{i}}^{2} \underline{m}_{i}}\right),
$$

where $\Gamma(\alpha)=\int_{0}^{+\infty} t^{\alpha-1} e^{-t} d t$ is the Gamma function and where $\mathbb{1}_{] 0,+\infty[}(\mu)$ is the indicator function. The parameter $\delta_{M_{i}}$ is such that $0 \leq \delta_{M_{i}}<1 / \sqrt{2}$. Therefore, the PDF of the random mass is completely defined by the mean value $\underline{m}_{i}$ and by the dispersion parameter $\delta_{M_{i}}$. 
3.1.2 Construction of the PDF for the random position vector $\boldsymbol{R}_{0, i}$

In this subsection, the PDF of the random initial position vector $\mathbf{R}_{0, i}$ of the center of mass of $\mathrm{RB}_{i}$ at initial time $t=0$ is constructed. In a first step, this available information is deduced from physical considerations. In a second step, the PDF of $\mathbf{R}_{0, i}$ is constructed by maximizing the entropy. In a third step, we develop a generator of independent realizations which will be useful for solving the stochastic dynamical equations using the Monte Carlo simulation method.

\section{(i) Available information}

The position vector $\underline{\mathbf{r}}_{0, i}$ (at initial time) of the center of mass $\underline{\mathcal{G}}_{i}$ of the nominal model is given. However, the real position is not exactly known and $\underline{\mathbf{r}}_{0, i}$ only corresponds to a mean position. Consequently, there is an uncertainty about the real position and this is the reason why this position is modeled by the random vector $\mathbf{R}_{0, i}$. In practice, the uncertain position vector of the center of mass cannot take any value as explained hereinafter. Some geometrical and mechanical considerations lead us to introduce an admissible domain $\mathcal{D}_{i}$ of random vector $\mathbf{R}_{0, i}$. Let $\mathbf{r}_{b, i}$ be the position vector of the barycenter of domain $\mathcal{D}_{i}$ defined as $\mathbf{r}_{b, i}=\left|\mathcal{D}_{i}\right|^{-1} \int_{\mathcal{D}_{i}} \mathbf{x} d \mathbf{x}$ in which $\left|\mathcal{D}_{i}\right|=\int_{\mathcal{D}_{i}} d \mathbf{x}$ is the volume of domain $\mathcal{D}_{i}$. In order to simplify the presentation, it is assumed that this subdomain $\mathcal{D}_{i}$ of $\mathbb{R}^{3}$ is completely defined by a vector-valued parameter $\mathbf{h}$. This means that as soon as $\mathbf{h}$ is fixed, then the geometry of the domain $\mathcal{D}_{i}$ is completely defined. Such a domain is then denoted by $\mathcal{D}_{i}(\mathbf{h})$ and below, $\mathbf{h}$ is either fixed or is an unknown deterministic vector-valued parameter which must be identified. In practice, $\mathbf{r}_{b, i}$ will be a part of the components of $\mathbf{h}$. For example, if $\mathcal{D}_{i}(\mathbf{h})$ is a ball, then vector-valued parameter $\mathbf{h}$ can be chosen as $\mathbf{h}=\left(\eta, \mathbf{r}_{b, i}\right)$ depending on the radius $\eta$ of the ball and of the center of the ball which represented by the vector $\mathbf{r}_{b, i}$. In addition, the mean value of the random vector $\mathbf{R}_{0, i}$ must be equal to the value $\underline{\mathbf{r}}_{0, i}$ of the nominal model. Therefore, the available information for the random variable $\mathbf{R}_{0, i}$ can be written as

$$
\begin{gathered}
\mathbf{R}_{0, i} \in \mathcal{D}_{i}(\mathbf{h}), \\
E\left\{\mathbf{R}_{0, i}\right\}=\underline{\mathbf{r}}_{0, i} \in \mathcal{D}_{i}(\mathbf{h}) .
\end{gathered}
$$

(ii) Maximum entropy principle

The probability density function $\mathbf{a} \mapsto p_{\mathbf{R}_{0, i}}(\mathbf{a})$ of random variable $\mathbf{R}_{0, i}$ is then constructed by maximizing the entropy defined by

$$
S\left(p_{\mathbf{R}_{0, i}}\right)=-\int_{\mathbb{R}^{3}} p_{\mathbf{R}_{0, i}}(\mathbf{a}) \log \left(p_{\mathbf{R}_{0, i}}(\mathbf{a})\right) d \mathbf{a},
$$

with the constraints defined by the available information in Eq. (18). The solution of this optimization problem depends on two parameters which are $\underline{\mathbf{r}}_{0, i}$ and vector-valued parameter $\mathbf{h}$, and is such that

$$
p_{\mathbf{R}_{0, i}}(\mathbf{a} ; \mathbf{h})=\mathbb{1}_{\mathcal{D}_{i}(\mathbf{h})}(\mathbf{a}) C_{0} e^{-<\boldsymbol{\lambda}_{r}, \mathbf{a}>},
$$

where $\langle\mathbf{a}, \mathbf{b}\rangle=a_{1} b_{1}+a_{2} b_{2}+a_{3} b_{3}$ denotes the Euclidean inner product of $\mathbf{a}$ with b. The positive valued parameter $C_{0}$ and vector $\boldsymbol{\lambda}_{r}$ are the unique solution of the equations

$$
\begin{aligned}
C_{0} \int_{\mathcal{D}_{i}(\mathbf{h})} e^{-<\boldsymbol{\lambda}_{r}, \mathbf{a}>} d \mathbf{a}=1 \\
C_{0} \int_{\mathcal{D}_{i}(\mathbf{h})} \mathbf{a} e^{-<\boldsymbol{\lambda}_{r}, \mathbf{a}>} d \mathbf{a}=\underline{\mathbf{r}}_{0, i}
\end{aligned}
$$


The equation (21-a) is relative to the normalization of the PDF and Eq. (21-b) is relative to the constraint defined by Eq. (18-b). Using Eq. (21-a), the parameter $C_{0}$ can be eliminated. Therefore, $\lambda_{\boldsymbol{r}}$ is solution of the following equation

$$
\int_{\mathcal{D}_{i}(\mathbf{h})} \mathbf{a} e^{-<\boldsymbol{\lambda}_{r}, \mathbf{a}>} d \mathbf{a}=\underline{\mathbf{r}}_{0, i} \int_{\mathcal{D}_{i}(\mathbf{h})} e^{-<\boldsymbol{\lambda}_{r}, \mathbf{a}>} d \mathbf{a}
$$

In the general case, the PDF of the random variable $\mathbf{R}_{0, i}$ is given by Eq. (20) for which vector $\boldsymbol{\lambda}_{r}$ is the unique solution of Eq. (22) and then, the constant $C_{0}$ is given solving Eq. (21-a).

Let us now consider the following particular case. Taking $\boldsymbol{\lambda}_{r}=\mathbf{0}$ in Eq. (22) yields $\mathbf{r}_{b, i}=\underline{\mathbf{r}}_{0, i}$ which means that the barycenter of the domain $\mathcal{D}_{i}(\mathbf{h})$ coincides with the position vector of the center of mass $\underline{\mathcal{G}}_{i}$ at initial time $t=0$ of the nominal model. Then using Eqs. (20) and (21-a) yields

$$
p_{\mathbf{R}_{0, i}}(\mathbf{a} ; \mathbf{h})=\frac{1}{\left|\mathcal{D}_{i}(\mathbf{h})\right|} \mathbb{1}_{\mathcal{D}_{i}(\mathbf{h})}(\mathbf{a})
$$

Since the solution of Eq. (21) is unique, it can be concluded that, if $\mathbf{r}_{b, i}=\underline{\mathbf{r}}_{0, i}$, then random variable $\mathbf{R}_{0, i}$ is uniform on $\mathcal{D}_{i}(\mathbf{h})$.

(iii) Generator of independent realizations

The independent realizations of random variable $\mathbf{R}_{0, i}$ must be generated using the constructed PDF $p_{\mathbf{R}_{0, i}}$. There are two cases.

(1) if $\mathbf{r}_{b, i}=\underline{\mathbf{r}}_{0, i}$ (case for which $\boldsymbol{\lambda}_{r}=\mathbf{0}$ ), then we have to generate independent realizations of a uniform random variable on the bounded subspace $\mathcal{D}_{i}(\mathbf{h})$ of $\mathbb{R}^{3}$. Let $\mathcal{V}$ be a cube in $\mathbb{R}^{3}$ such that $\mathcal{D}_{i}(\mathbf{h}) \subset \mathcal{V}$. Let $\mathbf{V}$ be the uniform $\mathbb{R}^{3}$-valued random variable on $\mathcal{V}$ for which the PDF is written as $p_{\mathbf{v}}(\mathbf{v})=|\mathcal{V}|^{-1} \mathbb{1}_{\mathcal{V}}(\mathbf{v})$. So it is very easy to generate independent realizations of random variable $\mathbf{V}$. If $\mathbf{a} \mapsto \mathbf{g}(\mathbf{a})$ is any (measurable) function, then it can be verified that

$$
E\left\{\mathbf{g}\left(\mathbf{R}_{0, i}\right)\right\}=\frac{|\mathcal{V}|}{\left|\mathcal{D}_{i}(\mathbf{h})\right|} E\left\{\mathbf{g}(\mathbf{V}) \frac{\mathbb{1}_{\mathcal{D}_{i}(\mathbf{h})}(\mathbf{V})}{\mathbb{1}_{\mathcal{V}}(\mathbf{V})}\right\},
$$

which is a general formula to estimate $E\left\{\mathbf{g}\left(\mathbf{R}_{0, i}\right)\right\}$ using the generator of $\mathbf{V}$.

(2) if $\mathbf{r}_{b, i} \neq \underline{\mathbf{r}}_{0, i}$ (case for which $\boldsymbol{\lambda}_{r} \neq \mathbf{0}$ ), then we have to generate independent realizations using the PDF defined by Eqs. (20) and (21). Such a generator can be obtained using the Monte Carlo Markov Chain (MCMC) method (Metropolis-Hastings algorithm [8] which is summarized in D).

\subsubsection{Random matrix $\left[\boldsymbol{J}_{i}\right]$ of the random tensor of inertia.}

In this subsection, the random matrix $\left[\mathbf{J}_{i}\right]$ of the random tensor of inertia with respect to $\left(\mathcal{G}_{i}, \underline{x}_{i, 1}^{\prime}, \underline{x}_{i, 2}^{\prime}, \underline{x}_{i, 3}^{\prime}\right)$ is defined and an algebraic representation of this random matrix is constructed. The mass distribution around the random center of mass $\mathcal{G}_{i}$ is uncertain and consequently, the tensor of inertia is also uncertain. This is the reason why the matrix $\left[\underline{J}_{i}\right]$ of the tensor of inertia of the nominal model with respect to $\left(\underline{\mathcal{G}}_{i}, \underline{x}_{i, 1}^{\prime}, \underline{x}_{i, 2}^{\prime}, \underline{x}_{i, 3}^{\prime}\right)$ is replaced by a random matrix $\left[\mathbf{J}_{i}\right]$ which is constructed by using 
the maximum entropy principle.

In a first step, we derive the fundamental properties for a tensor of inertia in order to propose a construction of the random matrix $\left[\mathbf{J}_{i}\right]$ which must be physically consistent. This will lead us to introduce the random matrix $\left[\mathbf{Z}_{i}\right]$ relative to the second-order moment of inertia of $\mathrm{RB}_{i}$. Then, in a second step, the available information is not directly constructed for the random matrix $\left[\mathbf{J}_{i}\right]$ but is constructed for the random matrix $\left[\mathbf{Z}_{i}\right]$. In the third step, the entropy is maximized under the constraints defined by the available information. In a fourth step, we derive the properties of the random matrix $\left[\mathbf{J}_{i}\right]$ which depends on the random matrix $\left[\mathbf{Z}_{i}\right]$ and on the random mass $M_{i}$. In a last step, a generator of independent realizations of the random matrix $\left[\mathbf{J}_{i}\right]$ is developed.

(i) Construction of the random matrix $\left[\boldsymbol{J}_{i}\right]$

In order to define the available information for the construction of the probability model of $\left[\mathbf{J}_{i}\right]$, we begin introducing two properties of the matrix $\left[J_{i}\right]$ defined by Eq. (3).

(1) Matrix $\left[J_{i}\right]$ defined by Eq. (3) depends on the mass $m_{i}$ through the mass density $\rho(\mathbf{x})$. Since $m_{i}$ is uncertain and modeled by the random variable $M_{i}$, the random variables $\left[\mathbf{J}_{i}\right]$ and $M_{i}$ are not independent. Therefore, it is important to normalize mass density $\rho(\mathbf{x})$ with respect to total mass $m_{i}$ for the construction of the probability model of the tensor of inertia. We then introduce $\left[\widetilde{J}_{i}\right]$ such that $\left[\widetilde{J}_{i}\right]=\left[J_{i}\right] / m_{i}$ and we have to construct the probability model $\left[\mathbf{J}_{i}\right]$ of $\left[\widetilde{J}_{i}\right]$. It should be noted that $\left[\widetilde{J}_{i}\right]$ depends on the normalized distribution of mass $\rho(\mathbf{x}) / m_{i}$, but is independent of the total mass $m_{i}$ and is independent of the center of mass $\mathbf{r}_{0, i}$ because $\left[J_{i}\right]$ is reduced at the center of mass.

(2) Matrix $\left[J_{i}\right]$ of the tensor of inertia is positive definite but verifies a stronger property which implies the positive definiteness and that we construct below. For all $\mathbf{u}$ in $\mathbb{R}^{3}$, the vector $\left[J_{i}\right] \mathbf{u}$ defined by Eq. (3) can be rewritten as

$$
\left[J_{i}\right] \mathbf{u}=\int_{\Omega_{i}}<\mathbf{x}^{\prime}, \mathbf{x}^{\prime}>\mathbf{u} \rho\left(\mathbf{x}^{\prime}\right) d \mathbf{x}^{\prime}-\int_{\Omega_{i}}<\mathbf{x}^{\prime}, \mathbf{u}>\mathbf{x}^{\prime} \rho\left(\mathbf{x}^{\prime}\right) d \mathbf{x}^{\prime} .
$$

We then have

$$
\left[J_{i}\right]_{j k}=\int_{\Omega_{i}} x_{\ell}^{\prime} x_{\ell}^{\prime} \delta_{j k} \rho\left(\mathbf{x}^{\prime}\right) d \mathbf{x}^{\prime}-\int_{\Omega_{i}} x_{j}^{\prime} x_{k}^{\prime} \rho\left(\mathbf{x}^{\prime}\right) d \mathbf{x}^{\prime}
$$

in which $\delta_{j k}$ is the Kronecker symbol and where the convention for summations over repeated Latin indices is used. Therefore, the trace of $\left[J_{i}\right]$ is such that $\operatorname{tr}\left(\left[J_{i}\right]\right)=$ $2 \int_{\Omega_{i}} x_{\ell}^{\prime} x_{\ell}^{\prime} \rho\left(\mathbf{x}^{\prime}\right) d \mathbf{x}^{\prime}$. We then have

$$
\left[J_{i}\right]=\frac{\operatorname{tr}\left(\left[J_{i}\right]\right)}{2}\left[I_{3}\right]-\left[H_{i}\right] \quad,
$$

where $\left[H_{i}\right]$ is the positive definite matrix of the second order tensor of inertia defined by

$$
\left[H_{i}\right](\mathbf{u})=\int_{\Omega_{i}}<\mathbf{x}^{\prime}, \mathbf{u}>\mathbf{x}^{\prime} \rho\left(\mathbf{x}^{\prime}\right) d \mathbf{x}^{\prime} \quad, \quad \forall \mathbf{u} \in \mathbb{R}^{3} .
$$

Taking the trace of Eq. (25) yields $\operatorname{tr}\left(\left[J_{i}\right]\right)=2 \operatorname{tr}\left(\left[H_{i}\right]\right)$ and substituting this equation into Eq. (25) yields

$$
\left[J_{i}\right]=\operatorname{tr}\left(\left[H_{i}\right]\right)\left[I_{3}\right]-\left[H_{i}\right]
$$


It can easily be shown that for dimension $n$ greater than 1

$$
[A] \in \mathbb{M}_{n}^{+}(\mathbb{R}) \Rightarrow\left\{\operatorname{tr}([A])\left[I_{3}\right]-[A]\right\} \in \mathbb{M}_{n}^{+}(\mathbb{R})
$$

The converse is false. Then applying this property to the matrix $[A]=\left[H_{i}\right]$ in Eq. (27) yields $\left[J_{i}\right]$ is positive definite. Therefore, the property $\left[H_{i}\right]$ is positive definite implies $\left[J_{i}\right]$ is positive definite. From Eq. $(25)$, it can be deduced that $\left[H_{i}\right]=\operatorname{tr}\left(\left[J_{i}\right]\right)\left[I_{3}\right] / 2-\left[J_{i}\right]$. Consequently, the property $\operatorname{tr}\left(\left[J_{i}\right]\right)\left[I_{3}\right] / 2-\left[J_{i}\right]$ is positive definite implies $\left[J_{i}\right]$ is positive definite (that is a consequence). Note that this property is stronger than the property $\left[J_{i}\right]$ is positive definite because, $\left[J_{i}\right]$ is positive definite does not imply that $\operatorname{tr}\left(\left[J_{i}\right]\right)\left[I_{3}\right] / 2-\left[J_{i}\right]$ is positive definite. Conversely, it is proven in Appendix A, that each positive definite matrix $\left[J_{i}\right]$ constructed using Eq. $(27)$, where $\left[H_{i}\right]$ is a given positive definite matrix, can be interpreted as the matrix of a tensor of inertia of a physical rigid body for which the mass is $m_{i}$. For summarizing, the available information concerning matrix $\left[J_{i}\right]$ is then $\operatorname{tr}\left(\left[J_{i}\right]\right)\left[I_{3}\right] / 2-\left[J_{i}\right]$ is positive definite (that implies $\left[J_{i}\right]$ is positive definite).

(3) Finally, developments (1) and (2) above lead us to introduce the positive-definite matrix $\left[Z_{i}\right]$ independent of $m_{i}$ such that

$$
\left[Z_{i}\right]=\frac{1}{m_{i}}\left\{\frac{\operatorname{tr}\left(\left[J_{i}\right]\right)}{2}\left[I_{3}\right]-\left[J_{i}\right]\right\},
$$

which yields $\left[J_{i}\right]=\operatorname{tr}\left(\left[J_{i}\right]\right)\left[I_{3}\right] / 2-m_{i}\left[Z_{i}\right]$. Taking the trace of this equation yields $\operatorname{tr}\left(\left[J_{i}\right]\right)=2 m_{i} \operatorname{tr}\left(\left[Z_{i}\right]\right)$. Substituting this equation into the previous one allows $\left[J_{i}\right]$ to be calculated as a function of $\left[Z_{i}\right]$,

$$
\left[J_{i}\right]=m_{i}\left\{\operatorname{tr}\left(\left[Z_{i}\right]\right)\left[I_{3}\right]-\left[Z_{i}\right]\right\} \quad .
$$

The probabilistic modeling $\left[\mathbf{J}_{i}\right]$ of $\left[J_{i}\right]$ consists in introducing the random matrix $\left[\mathbf{Z}_{i}\right]$ and in using Eq. (30) in which $m_{i}$ is replaced by the random variable $M_{i}$ and where $\left[Z_{i}\right]$ is replaced by $\left[\mathbf{Z}_{i}\right]$. We then obtain

$$
\begin{aligned}
& {\left[\mathbf{Z}_{i}\right]=\frac{1}{M_{i}}\left\{\frac{\operatorname{tr}\left(\left[\mathbf{J}_{i}\right]\right)}{2}\left[I_{3}\right]-\left[\mathbf{J}_{i}\right]\right\},} \\
& {\left[\mathbf{J}_{i}\right]=M_{i}\left\{\operatorname{tr}\left(\left[\mathbf{Z}_{i}\right]\right)\left[I_{3}\right]-\left[\mathbf{Z}_{i}\right]\right\} .}
\end{aligned}
$$

(ii) Available information concerning the random matrix $\left[\boldsymbol{Z}_{i}\right]$

We then have to define the available information relative to the random matrix $\left[\mathbf{Z}_{i}\right]$ in order to construct its probability distribution using the maximum entropy principle. We have proven that the deterministic matrix $\left[Z_{i}\right]$ is positive definite. Consequently, its stochastic model $\left[\mathbf{Z}_{i}\right]$ must be a random matrix with values in $\mathbb{M}_{3}^{+}(\mathbb{R})$. We will say that a matrix $[A]$ is less that a matrix $[B]$, that is to say that $[A]<[B]$, if $[B]-[A]$ is a positive definite matrix. Since the bounded domain $\Omega_{i}$ is fixed, the deterministic matrix $\left[Z_{i}\right]$ is less than the matrix $\left[Z_{i}^{\max }\right]$ defined by

$$
\left[Z_{i}^{\max }\right]_{j k}=\max \left(x_{j}^{\prime 2}\right) \delta_{j k}
$$

in which $\max \left(x_{j}^{\prime}{ }^{2}\right)$ is the maximum value of ${x_{j}^{\prime}}^{2}$ in the domain $\Omega_{i}$. Finally, the constraints relative to the support of the probability distribution of the random matrix 
$\left[\mathbf{Z}_{i}\right]$ are $\left[\mathbf{Z}_{i}\right] \in \mathbb{M}_{3}^{+}(\mathbb{R})$ and $\left[Z_{i}^{\max }\right]-\left[\mathbf{Z}_{i}\right] \in \mathbb{M}_{3}^{+}(\mathbb{R})$. Let us introduce the nominal value $\left[\underline{Z}_{i}\right]$ of deterministic matrix $\left[Z_{i}\right]$ such that

$$
\left[\underline{Z}_{i}\right]=\frac{1}{\underline{m}_{i}}\left\{\frac{\operatorname{tr}\left(\left[\underline{J}_{i}\right]\right)}{2}\left[I_{3}\right]-\left[\underline{J}_{i}\right]\right\} .
$$

By construction, the mean value of the random matrix $\left[\mathbf{Z}_{i}\right]$ is equal to $\left[\underline{Z}_{i}\right]$. We also introduce the inequality $\left|E\left\{\log \left(\operatorname{det}\left[\mathbf{Z}_{i}\right]\right)\right\}\right|<+\infty$ which will be useful to obtain a second-order stochastic solution of the nonlinear dynamical system and the inequality $\left|E\left\{\log \left(\operatorname{det}\left(\left[Z_{i}^{\max }\right]-\left[\mathbf{Z}_{i}\right]\right)\right)\right\}\right|<+\infty$ which allows the upper bound $\left[Z_{i}^{\max }\right]$ to be reached with a zero probability. Consequently, the available information for $\left[\mathbf{Z}_{i}\right]$ can be summarized as follows,

$$
\begin{gathered}
{\left[\mathbf{Z}_{i}\right] \in \mathbb{M}_{3}^{+}(\mathbb{R}),} \\
\left\{\left[Z_{i}^{\max }\right]-\left[\mathbf{Z}_{i}\right]\right\} \in \mathbb{M}_{3}^{+}(\mathbb{R}), \\
E\left\{\left[\mathbf{Z}_{i}\right]\right\}=\left[\underline{Z}_{i}\right], \\
E\left\{\log \left(\operatorname{det}\left[\mathbf{Z}_{i}\right]\right)\right\}=C_{i}^{l}, \quad\left|C_{i}^{l}\right|<+\infty . \\
E\left\{\log \left(\operatorname{det}\left(\left[Z_{i}^{\max }\right]-\left[\mathbf{Z}_{i}\right]\right)\right)\right\}=C_{i}^{u}, \quad\left|C_{i}^{u}\right|<+\infty .
\end{gathered}
$$

For more convenience, the random matrix $\left[\mathbf{Z}_{i}\right]$ is normalized as follow. Matrix $\left[\underline{Z}_{i}\right]$ being positive definite, its Cholesky decomposition yields

$$
\left[\underline{Z}_{i}\right]=\left[\underline{L}_{Z_{i}}\right]^{T}\left[\underline{L}_{Z_{i}}\right],
$$

in which $\left[\underline{L}_{Z_{i}}\right]$ is an upper triangular matrix in the set $\mathbb{M}_{3}(\mathbb{R})$ of all the $(3 \times 3)$ real matrices. Then, the random matrix $\left[\mathbf{Z}_{i}\right]$ can be rewritten as

$$
\left[\mathbf{Z}_{i}\right]=\left[\underline{L}_{Z_{i}}\right]^{T}\left[\mathbf{G}_{i}\right]\left[\underline{L}_{Z_{i}}\right],
$$

in which the matrix $\left[\mathbf{G}_{i}\right]$ is a random matrix for which the available information is

$$
\begin{gathered}
{\left[\mathbf{G}_{i}\right] \in \mathbb{M}_{3}^{+}(\mathbb{R}),} \\
\left\{\left[G_{i}^{\mathrm{max}}\right]-\left[\mathbf{G}_{i}\right]\right\} \in \mathbb{M}_{3}^{+}(\mathbb{R}), \\
E\left\{\left[\mathbf{G}_{i}\right]\right\}=\left[I_{3}\right] \quad, \\
E\left\{\log \left(\operatorname{det}\left[\mathbf{G}_{i}\right]\right)\right\}=C_{i}^{l^{\prime}}, \quad\left|C_{i}^{l^{\prime}}\right|<+\infty, \\
E\left\{\log \left(\operatorname{det}\left(\left[G_{i}^{\max }\right]-\left[\mathbf{G}_{i}\right]\right)\right)\right\}=C_{i}^{u \prime}, \quad\left|C_{i}^{u \prime}\right|<+\infty
\end{gathered}
$$

in which $C_{i}^{l^{\prime}}=C_{i}^{l}-\log \left(\operatorname{det}\left[\underline{Z}_{i}\right]\right), C_{i}^{u \prime}=C_{i}^{u}-\log \left(\operatorname{det}\left[\underline{Z}_{i}\right]\right)$ and where the matrix $\left[G_{i}^{\max }\right]$ is an upper bound for the random matrix $\left[\mathbf{G}_{i}\right]$ and is defined by

$$
\left[G_{i}^{\max }\right]=\left(\left[\underline{L}_{Z_{i}}\right]^{T}\right)^{-1}\left[Z_{i}^{\max }\right]\left[\underline{L}_{Z_{i}}\right]^{-1} .
$$

The maximum entropy principle is applied to the random matrix $\left[\mathbf{G}_{i}\right]$, and the random matrix $\left[\mathbf{Z}_{i}\right]$ is constructed using Eq. (37).

(iii) Maximum entropy principle

The probability distribution of the random matrix $\left[\mathbf{G}_{i}\right]$ is constructed using the maximum entropy principle under the constraints defined by the available information given 
by Eq. (38). If only constraints defined by Eqs. (38-a),(38-c) and (38-d) are taken into account we obtain the ensemble of normalized positive-definite symmetric random real matrices introduced in [28] and [29]. This ensemble was originally introduced to take into account both the system-parameter uncertainties and the model uncertainties induced by modeling errors in structural dynamics, yielding the so-called nonparametric probabilistic approach of uncertainties (see [28], [29], [30] for which experimental validations can be found, for instance, in [1], [4], [6] and [7]). Let $\left[\mathbf{G}_{0, i}\right]$ be the random matrix constructed with the constraints defined by Eqs. (38-a),(38-c) and (38-d). Then, the probability density function $p_{\left[\mathbf{G}_{0, i}\right]}([G])$ of the random matrix $\left[\mathbf{G}_{0, i}\right]$ with respect to the volume element $\widetilde{d} G=2^{3 / 2} \Pi_{1 \leq j \leq k \leq 3} d G_{j k}$ on the set $\mathbb{M}_{3}^{S}(\mathbb{R})$ of all the symmetric $(3 \times 3)$ real matrices is written as

$$
p_{\left[\mathbf{G}_{0, i}\right]}([G])=\mathbb{1}_{\mathbb{M}_{3}^{+}(\mathbb{R})}([G]) \times C_{G_{0, i}} \times(\operatorname{det}[G])^{-\lambda} \times e^{-\operatorname{tr}\left(\left[\mu_{0}\right][G]\right)},
$$

in which the positive valued parameter $C_{G_{0, i}}$ is a normalization constant, the real parameter $\lambda<1$ is a Lagrange multiplier relative to the constraint defined by Eq. (38-d) and the positive definite matrix $\left[\mu_{0}\right]$ is a Lagrange multiplier relative to the constraint defined by Eq. (38-c). If constraints defined Eqs. (38-b) and (38-e) are also taken into account, the probability density function $p_{\left[\mathbf{G}_{i}\right]}([G])$ with respect to the volume element $\widetilde{d} G$ of random matrix $\left[\mathbf{G}_{i}\right]$ is then written as

$$
\begin{aligned}
p_{\left[\mathbf{G}_{i}\right]}([G]) & =\mathbb{1}_{\mathbb{M}_{3}^{+}(\mathbb{R})}([G]) \times \mathbb{1}_{\mathbb{M}_{3}^{+}(\mathbb{R})}\left(\left[G_{i}^{\max }\right]-[G]\right) \times C_{G_{i}} \\
& \times(\operatorname{det}[G])^{-\lambda_{l}} \times\left(\operatorname{det}\left(\left[G_{i}^{\text {max }}\right]-[G]\right)\right)^{-\lambda_{u}} \times e^{-\operatorname{tr}([\mu][G])},
\end{aligned}
$$

in which the positive valued parameter $C_{G_{i}}$ is a normalization constant, the real parameters $\lambda_{l}<1$ and $\lambda_{u}<1$ are Lagrange multipliers relative to the constraints defined by Eqs. (38-d) and (38-e) and the symmetric real matrix $[\mu]$ is a Lagrange multiplier relative to the constraint defined by Eq. (38-c). This probability density function is a particular case the Kummer-Beta matrix variate distribution (see [17], [5], [9]) for which the lower bound is a zero matrix. It should be noted that we have not taken into account a non zero lower bound for the random matrix $\left[\mathbf{Z}_{i}\right]$. Indeed, the construction of such a non zero lower bound (which should be reached with a zero probability) is very difficult because there is no available information from the design.

Parameters $C_{G_{i}}, \lambda_{l}, \lambda_{u}$ and matrix $[\mu]$ are the unique solution of the equations

$$
\begin{gathered}
E\left\{\mathbb{1}_{\mathbb{M}_{3}^{S}(\mathbb{R})}\left(\left[\mathbf{G}_{i}\right]\right)\right\}=1, \\
E\left\{\left[\mathbf{G}_{i}\right]\right\}=\left[I_{3}\right], \\
E\left\{\log \left(\operatorname{det}\left[\mathbf{G}_{i}\right]\right)\right\}=C_{i}^{l^{\prime}}, \\
E\left\{\log \left(\operatorname{det}\left(\left[G_{i}^{\max }\right]-\left[\mathbf{G}_{i}\right]\right)\right)\right\}=C_{i}^{u \prime} .
\end{gathered}
$$

In Eq. (42), the mathematical expectation corresponds to an integration (over a subset of dimension 6) which cannot explicitly be done and which can be evaluated using an adapted numerical method. These integrals could be estimated with the Monte Carlo simulation method which would require a generator of independent realizations corresponding to the PDF defined by Eq. (41). Unfortunately, such a generator cannot directly be constructed. Note that a Monte Carlo Markov Chain (MCMC) simulation method could be used, such as the Metropolis-Hastings algorithm [8] or the algorithm recently proposed in [31] and based on the Itô stochastic differential calculus. Taking into account the algebraic expression of the PDF defined by Eq. (41), a more efficient 
algorithm can be used. It consists in estimating the integrals defined in Eq. (42) by the Monte Carlo simulation method with the PDF defined by Eq. (40) and relative to random matrix $\left[\mathbf{G}_{i, 0}\right]$ for which a generator of independent realizations is known [28] (for readability of the paper, this generator is detailed in Appendix B). Let $u \mapsto \mathbf{g}(u)$ be any vector-valued function defined in $\mathbb{M}_{3}^{S}(\mathbb{R})$. Then, using Eqs. (40) and (41), it can be deduced that

$$
\begin{aligned}
E\left\{\mathbf{g}\left(\left[\mathbf{G}_{i}\right]\right\}=\right. & \frac{C_{G_{i}}}{C_{G_{0, i}}} E\left\{\mathbf { g } \left(\left[\mathbf{G}_{i, 0}\right] \mathbb{1}_{\mathbb{M}_{3}^{+}(\mathbb{R})}\left(\left[G_{i}^{\max }\right]-\left[\mathbf{G}_{i, 0}\right]\right)\right.\right. \\
& \left.\times\left(\operatorname{det}\left(\left[G_{i}^{\max }\right]-\left[\mathbf{G}_{i, 0}\right]\right)\right)^{-\lambda_{u}}\right\} .
\end{aligned}
$$

Then Eq. (43) allows integrals of Eq. (42) to be estimated using independent realizations of $\left[\mathbf{G}_{i, 0}\right]$ and the Monte Carlo simulation method.

For fixed values of $\lambda_{l}$ and $\lambda_{u}$, parameters $C_{G_{i}}$ and $[\mu]$ can be estimated using Eq. (42). In Eq. (42), since the parameters $C_{i}^{l^{\prime}}$ and $C_{i}^{u \prime}$ have no real physical meaning, the parameters $\lambda_{l}$ and $\lambda_{u}$ are kept as parameters which then allows the "shape" of the PDF to be controlled. If experimental data are available for the responses of the dynamical system, then the two parameters $\lambda_{l}$ and $\lambda_{u}$ can be identified solving an inverse problem. If experimental data are not available, these two parameters allow a sensitivity analysis of the solution to be carried out with respect to the level of uncertainties.

(iv) Properties for the random matrix $\left[\boldsymbol{J}_{i}\right]$

In Appendix C, it is proven that using Eq. (32) and the available information defined by Eq. (35), the following important properties for the random matrix $\left[\mathbf{J}_{i}\right]$ can be deduced,

$$
\begin{gathered}
\left\{\frac{1}{2} \operatorname{tr}\left(\left[\mathbf{J}_{i}\right]\right)\left[I_{3}\right]-\left[\mathbf{J}_{i}\right]\right\} \in \mathbb{M}_{3}^{+}(\mathbb{R}), \\
\left\{\left[\mathbf{J}_{i}^{\max }\right]-\left[\mathbf{J}_{i}\right]\right\} \in \mathbb{M}_{3}^{+}(\mathbb{R}), \\
E\left\{\left[\mathbf{J}_{i}\right]\right\}=\left[\underline{J}_{i}\right], \\
\lambda_{l}<-2 \Rightarrow E\left\{\left\|\left[\mathbf{J}_{i}\right]^{-1}\right\|^{2}\right\}<+\infty,
\end{gathered}
$$

in which the random matrix $\left[\mathbf{J}_{i}^{\max }\right]$, which represents a random upper bound for the random matrix $\left[\mathbf{J}_{i}\right]$, is defined by

$$
\left[\mathbf{J}_{i}^{\max }\right]=M_{i}\left\{\operatorname{tr}\left(\left[Z_{i}^{\max }\right]\right)\left[I_{3}\right]-\left[Z_{i}^{\max }\right]\right\} .
$$

It should be noted that Eq. (44-a) implies that each realization of the random matrix $\left[\mathbf{J}_{i}\right]$ corresponds to the matrix of a tensor of inertia of a physical rigid body. In addition, this equation implies that the random matrix $\left[\mathbf{J}_{i}\right]$ is positive definite. Eq. (44-b) provides a random upper bound for the random matrix $\left[\mathbf{J}_{i}\right]$. Eq. (44-c) corresponds to a construction for which the mean value of the random matrix $\left[\mathbf{J}_{i}\right]$ is equal to the nominal value $\left[\underline{J}_{i}\right]$. Finally, Eq. $(44-\mathrm{d})$ is necessary for that the random solution of the nonlinear dynamical system be a second-order stochastic process.

(v) Generator of independent realizations for the random matrix $\left[\boldsymbol{J}_{i}\right]$

The generator described above is used to identify parameters $C_{G_{i}}, \lambda_{l}, \lambda_{u}$ and $[\mu]$. The integrals defined in Eq. (42) are then estimated using the Monte Carlo simulation method with the PDF defined by Eq. (40) relative to random matrix $\left[\mathbf{G}_{0, i}\right]$. Such a method is very efficient. However, this method is not adapted to calculate the random responses statistics of the multibody dynamical system with random rigid bodies 
because the randomness of the responses is also due to the randomness of the the mass and of the center of mass and consequently, the convergence is very slow. For this reason, we introduce a second generator of independent realizations of the random matrix $\left[\mathbf{G}_{i}\right]$ based on the Monte Carlo Markov Chain (MCMC) (Metropolis-Hastings algorithm [8] which is summarized in Appendix D) with the PDF defined by Eq. (41). Then, independent realizations of the random matrix $\left[\mathbf{Z}_{i}\right]$ are obtained using Eq. (37). Finally, independent realizations of the random matrix $\left[\mathbf{J}_{i}\right]$ are obtained using Eq. (32) and independent realizations of the random mass $M_{i}$.

3.2 Stochastic matrix model for a multibody dynamical system with uncertain rigid bodies and its random response

In order to limit the developments, it is assumed that only one of the $n_{b}$ rigid bodies denoted by $\mathrm{RB}_{i}$ of the rigid multibody system is uncertain. The extension to several uncertain rigid bodies is straightforward. Let the $6 n_{b}$ random coordinates be represented by the $\mathbb{R}^{6 n_{b}}$-valued stochastic process $\mathbf{U}=\left(\mathbf{R}_{1}, \ldots, \mathbf{R}_{n_{b}}, \mathbf{S}_{1}, \ldots, \mathbf{S}_{n_{b}}\right)$ indexed by $[0, T]$ and let the $n_{c}$ random Lagrange multipliers be represented by the $\mathbb{R}^{n_{c}}$-valued stochastic process $\boldsymbol{\Lambda}$ indexed by $[0, T]$. The deterministic Eq. (13) becomes the following stochastic equation

$$
\begin{gathered}
{\left[\begin{array}{c}
{[\mathbf{M}]} \\
{\left[\begin{array}{c}
{\left[\boldsymbol{\varphi}_{\mathbf{u}}\right]} \\
{[0]}
\end{array}\right]^{T}}
\end{array}\right]\left[\begin{array}{c}
\ddot{\mathbf{U}} \\
\boldsymbol{\Lambda}
\end{array}\right]=\left[\begin{array}{c}
\mathbf{q}-\mathbf{K} \\
-\frac{d}{d t} \boldsymbol{\varphi}_{t}-\left[\frac{d}{d t} \boldsymbol{\varphi}_{\mathbf{u}}\right] \dot{\mathbf{U}}
\end{array}\right], \quad \forall t \in[0, T],} \\
\mathbf{U}(0)=\mathbf{U}_{0} \quad, \quad \dot{\mathbf{U}}(0)=\mathbf{v}_{0},
\end{gathered}
$$

in which the vector $\mathbf{U}_{0}=\left(\mathbf{r}_{0,1}, \ldots, \mathbf{R}_{0, i}, \ldots, \mathbf{r}_{0, n_{b}}, \mathbf{s}_{0,1}, \ldots, \mathbf{s}_{0, n_{b}}\right)$ is random due to the random vector $\mathbf{R}_{0, i}$. For all given real vector $\dot{\mathbf{u}}$, the vector $\mathbf{K}(\dot{\mathbf{u}})$ defined by

$$
\mathbf{K}(\dot{\mathbf{u}})=\left(\mathbf{0}, \ldots, \mathbf{0}, \mathbf{k}_{1}\left(\boldsymbol{\omega}_{1},\left[J_{1}\right]\right) \ldots, \mathbf{k}_{i}\left(\boldsymbol{\omega}_{i},\left[\mathbf{J}_{i}\right]\right), \ldots, \mathbf{k}_{n_{b}}\left(\boldsymbol{\omega}_{n_{b}},\left[J_{n_{b}}\right]\right)\right)
$$

is random due to the random matrix $\left[\mathbf{J}_{i}\right]$. For all given real vectors $\mathbf{u}$ and $\dot{\mathbf{u}}$, the vector $\mathbf{q}(\mathbf{u}, \dot{\mathbf{u}}, t)$ is defined, as previously, by

$$
\mathbf{q}(\mathbf{u}, \dot{\mathbf{u}}, t)=\left(\mathbf{f}_{1}^{e}(\mathbf{u}, \dot{\mathbf{u}}, t), \ldots, \mathbf{f}_{n_{b}}^{e}(\mathbf{u}, \dot{\mathbf{u}}, t), \mathbf{l}_{1}^{e}(\mathbf{u}, \dot{\mathbf{u}}, t), \ldots, \mathbf{l}_{n_{b}}^{e}(\mathbf{u}, \dot{\mathbf{u}}, t)\right)
$$

The random mass matrix $[\mathbf{M}]$ is defined by

$$
[\mathbf{M}]=\left[\begin{array}{cc}
{\left[\mathbf{M}^{r}\right]} & 0 \\
0 & {\left[\mathbf{M}^{s}\right]}
\end{array}\right],
$$

in which the $\left(3 n_{b} \times 3 n_{b}\right)$ random matrices $\left[\mathbf{M}^{r}\right]$ and $[\mathbf{M}]^{s}$ are defined by

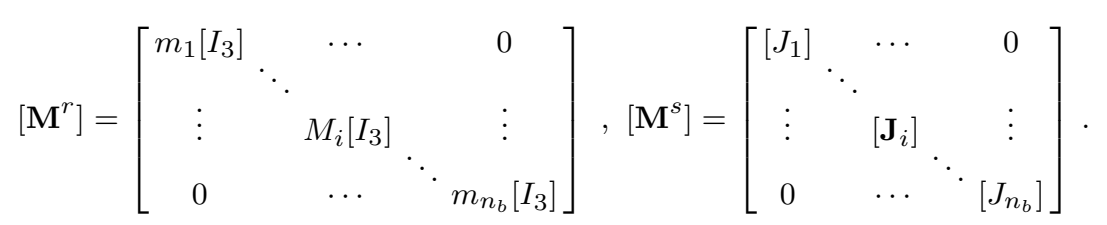

Random Eqs. (46) and (47) are solved using the Monte Carlo simulation method [19]. The level of dispersion of the random response is controlled by the dispersion parameter 
$\delta_{M_{i}}$ of the random mass, the geometric parameter $\mathbf{h}$ of the random initial position of the center of mass and the parameters $\lambda_{l}$ and $\lambda_{u}$ of the random inertia tensor. If experimental responses were available, then these four parameters could be identified using adapted mathematical statistics such as the maximum likelihood method [26] (see for instance [32], [1]). If no experimental responses are available, then these parameters can be used to analyse the sensitivity of the random solution with respect to the level of uncertainties, that is to say, to analyse the robustness of the model predictions with respect to uncertainties.

\section{Application}

In this section, we present a numerical application which validates the methodology presented in this paper.

\subsection{Description of the nominal model}

The rigid multibody model is made up of five rigid bodies and six joints which are described in the fixed frame $\left(O, x_{0,1}, x_{0,2}, x_{0,3}\right)$ (see Fig. 1). The plan defined by $\left(O, x_{0,1}, x_{0,2}\right)$ is identified below as the "ground". The gravity forces in the $x_{0,3^{-}}$ direction are taken into account.

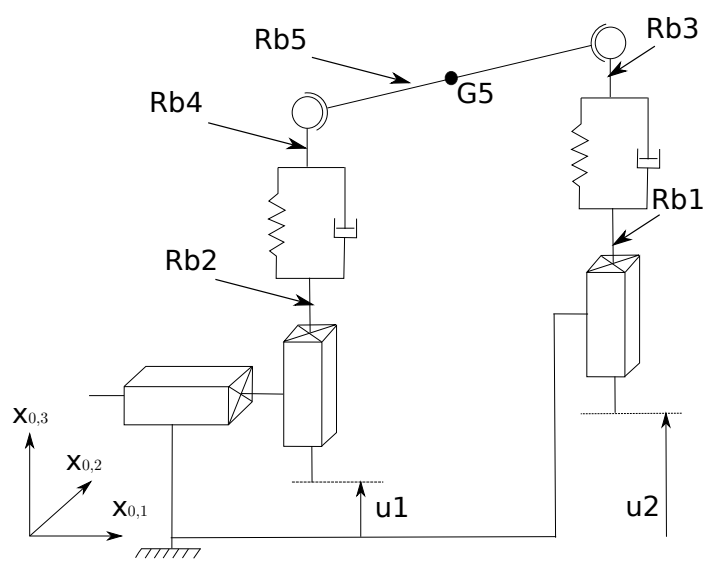

Fig. 1 Rigid multibody system.

\section{(i) Rigid bodies}

In the initial configuration, the rigid bodies $R b 1, R b 2, R b 3$ and $R b 4$ are cylinders for which the axes follow the $x_{0,3}$-direction and for which the radius is $0.02 \mathrm{~m}$, the length is $0.2 \mathrm{~m}$ and the mass density is $5013 \mathrm{~kg} / \mathrm{m}^{3}$. The initial position of the center of mass for rigid bodies $R b 1, R b 2, R b 3$ and $R b 4$ are respectively $(2.5,0,0),(-2.5,0,0),(2.5,0,0.3)$ and $(-2.5,0,0.3)$. In the initial configuration, the rigid body $R b 5$ is supposed to be symmetric with respect to the planes $\left(\mathcal{G} 5, x_{0,1}, x_{0,2}\right)$ and $\left(\mathcal{G} 5, x_{0,1}, x_{0,3}\right)$ in which $\mathcal{G} 5$ is the center of mass of $R b 5$ for which the initial position is $\underline{\mathbf{r}}_{0,5}=(0.6,0.0,0.5)$. The mass 
of $R b 5$ is $\underline{m}_{5}=3000 \mathrm{~kg}$. The tensor of inertia of $R b 5$ in the frame $\left(\mathcal{G} 5, x_{0,1}, x_{0,2}, x_{0,3}\right)$ is

$$
\underline{J}_{5}=\left[\begin{array}{ccc}
1200 & 0 & 0 \\
0 & 5000 & 0 \\
0 & 0 & 6000
\end{array}\right]
$$

\section{(ii) Joints}

- The joint Ground-Rb1 is made up of a prismatic joint following $x_{0,3}$-direction. The displacement following $x_{0,3}$-direction (see Fig. 1 ), denoted by $u 1(t)$, is imposed. The joint Ground-Rb2 is a prismatic joint following $x_{0,3}$-direction. The displacement following $x_{0,3}$-direction (see Fig. 1 ), denoted by $u 2(t)$, is imposed. The displacement following $x_{0,1}$-direction is unconstrained. Imposed displacements $u 1(t)$ and $u 2(t)$ are plotted in Fig. 2 and Fig. 3 for $t$ in $[0,0.03] s$.

- The joints $R b 1-R b 3$ and $R b 2-R b 4$ are constituted of $6 \mathrm{D}$ spring-dampers. For the three displacements and the three rotations, the matrix of the spring- and damperconstitutive equations is diagonal. The displacement stiffness is $1.0 \times 10^{9} \mathrm{~N} / \mathrm{m}$ for the three directions. The rotation stiffness is $1.6 \times 10^{8} \mathrm{Nm} / \mathrm{rad}$ for the three directions. The displacement and the rotation dampings are $2.4 \times 10^{6} \mathrm{Ns} / \mathrm{m}$ and $1.3 \times 10^{6} \mathrm{Nm} / \mathrm{rad}$, respectively, in the three directions. In each spring, the initial prestress due to the weight of the rigid body $R b 5$ is $(0,0,-14715 N)$.

- Finally, the joints $R b 3-R b 5$ and $R b 4-R b 5$ are $x_{0,2}$-direction revolute joints localized at points $(2.5,0,0.5)$ and $(-2.5,0,0.5)$, respectively.

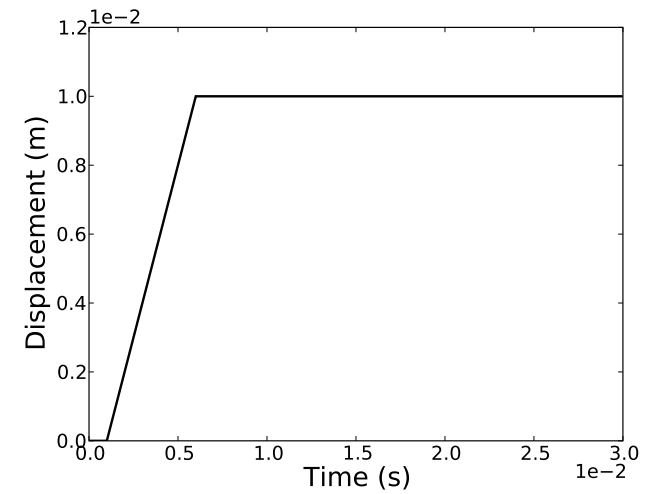

Fig. 2 Imposed displacement $u 1(t)$. 


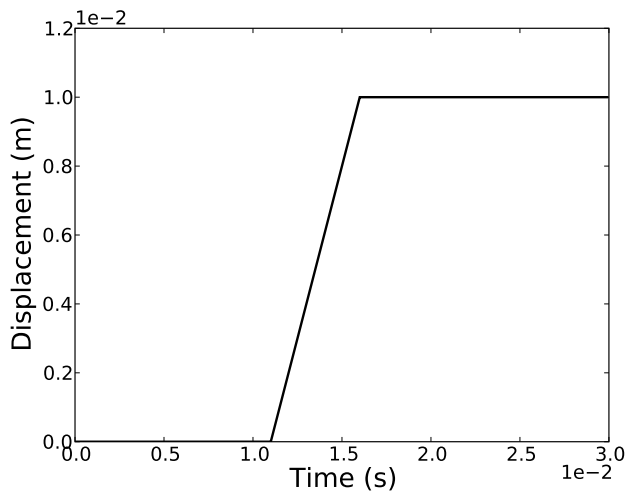

Fig. 3 Imposed displacement $u 2(t)$

4.2 Response of the nominal model

The initial velocities and angular velocities are zero. The transient response is calculated using the implicit multistep integration scheme presented in [2] (implemented in the software MBDyn which has been used for the simulations) with a time step $\Delta t=1.0 \times 10^{-5} \mathrm{~s}$. The observation point $P_{o b s}$ belongs to $R b 5$ and is initially located at position $(-2.0,-1.0,0.5)$. The displacements and rotations (Euler angles in the sequence $(1,2,3))$ of point $P_{\text {obs }}$ are plotted in Figs. $4-9$ for $t$ in $[0,0.1] s$. We can remark that the motion occurs in the plane $\left(P_{o b s}, x_{0,1}, x_{0,3}\right)$ because of the symmetries of the problem. These symmetries will be broken when introducing uncertainties in the tensor of inertia and initial position of the center of mass for rigid body $R b 5$. Below, we are only interested in the transient acceleration of point $P_{o b s}$ for $t$ in $[0,0.03] s$ (for the which the permanent response is not reached yet).

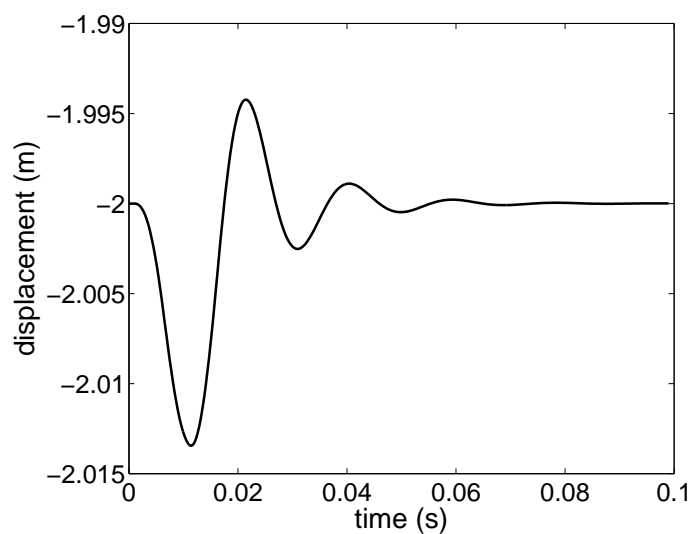

Fig. 4 Transient response of point $P_{o b s}: x_{0,1}$-displacement. 


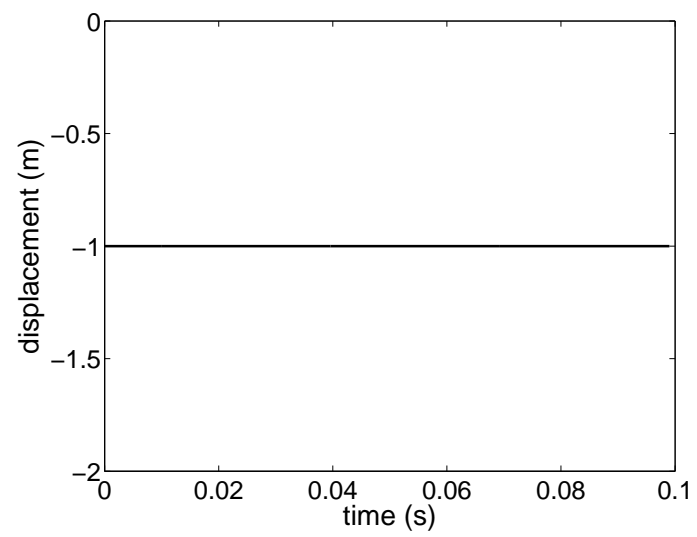

Fig. 5 Transient response of point $P_{o b s}: x_{0,2}$-displacement.

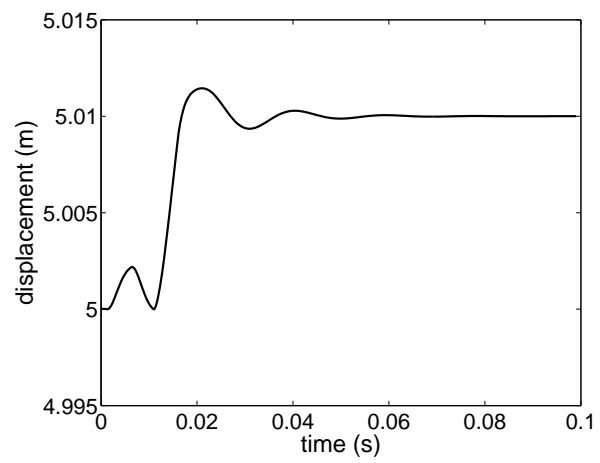

Fig. 6 Transient response of point $P_{o b s}: x_{0,3}$-displacement. 


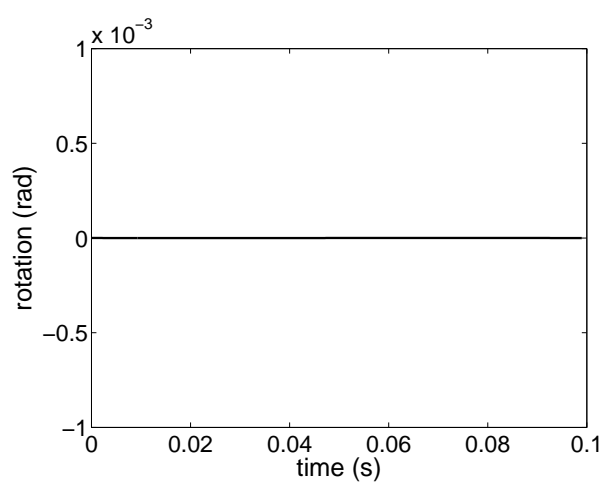

Fig. 7 Transient response of point $P_{o b s}: x_{0,1}$-rotation.

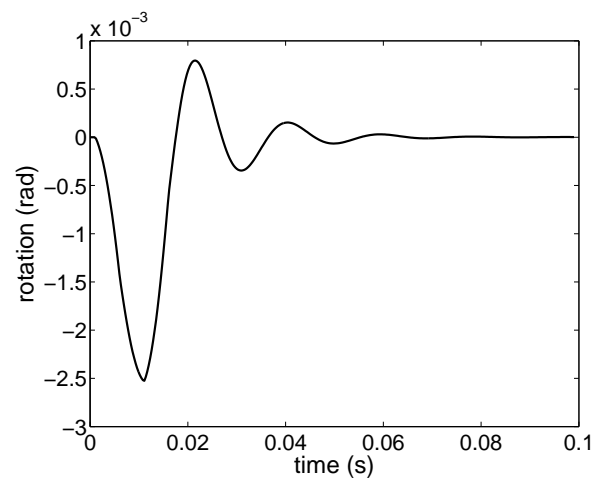

Fig. 8 Transient response of point $P_{o b s}: x_{0,2}$-rotation. 


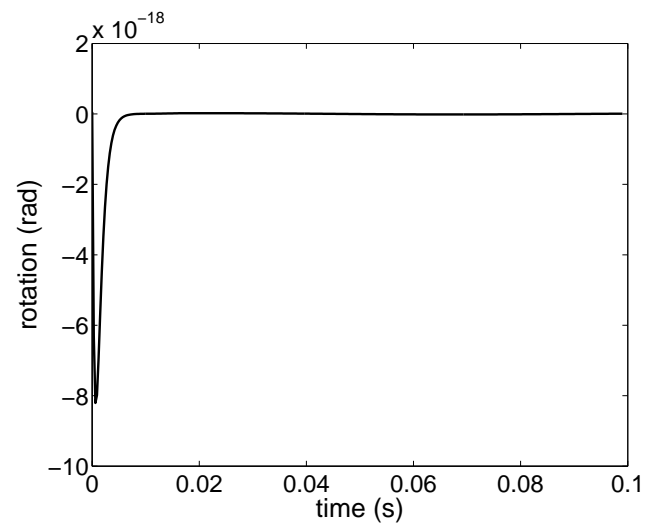

Fig. 9 Transient response of point $P_{o b s}: x_{0,3}$-rotation.

4.3 Random response of the stochastic model

Rigid body $R b 5 a$ is considered as uncertain and is therefore modeled by a random rigid body. As explained in Section 3, the elements of inertia of the uncertain rigid body $R b 5$ are replaced by random quantities. The fluctuation of the response is controlled by four parameters $\delta_{M_{5}}, \mathbf{h}, \lambda_{l}$ and $\lambda_{u}$. A sensitivity analysis is carried out with respect to these four parameters. The statistics for the transient responses are estimated using the Monte Carlo simulation method with 500 independent realizations. This number of realizations has been determined in order to get a good mean-square convergence of the stochastic responses.

(i) Case 1: $M_{5}$ is random, $\mathbf{R}_{0,5}$ is deterministic and $\left[\mathbf{J}_{5}\right]$ is deterministic.

We choose $\delta_{M_{5}}=0.5$. The probability density function of random mass $M_{i}$ is plotted in Fig. 10. The confidence region, with a probability level $P_{c}=0.90$, of the random acceleration of point $P_{o b s}$ is plotted in Figs. 11-16. It can be noted that all non-zero accelerations are sensitive to the mass uncertainties. It can also be viewed that all angular accelerations are sensitive to the mass uncertainties. Indeed, the tensor of inertia depends on the random mass $M_{5}$ through Eq. (32). 


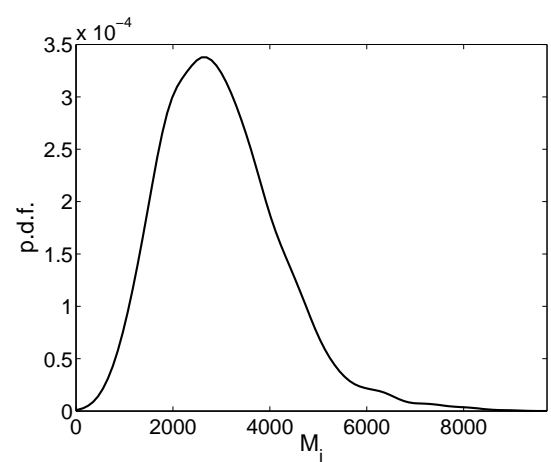

Fig. 10 Probability density function of random mass $M_{5}$

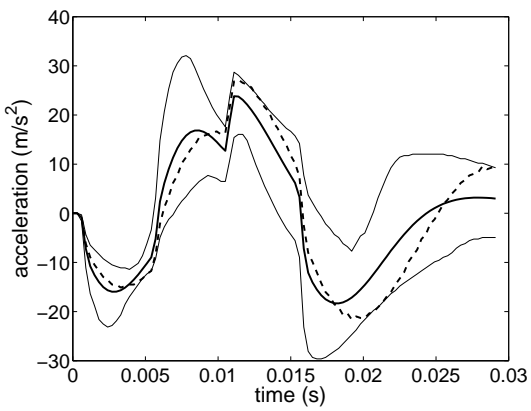

Fig. 11 Random transient acceleration of point $P_{o b s}$, Case 1: confidence region (upper and lower thin solid lines), mean response (thick solid line) and response of the nominal model (dashed line); $x_{0,1}$-acceleration.

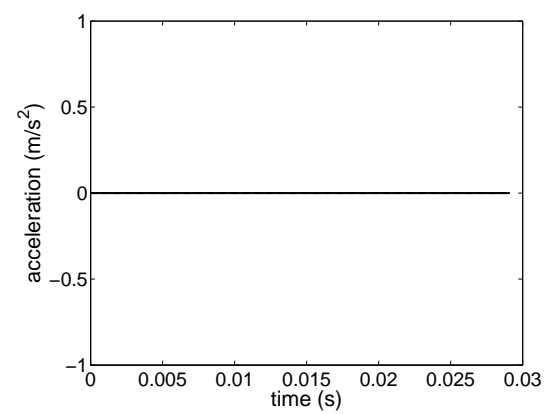

Fig. 12 Random transient acceleration of point $P_{o b s}$, Case 1: confidence region (upper and lower envelopes), mean response and response of the nominal model are superimposed; $x_{0,2^{-}}$ acceleration. 


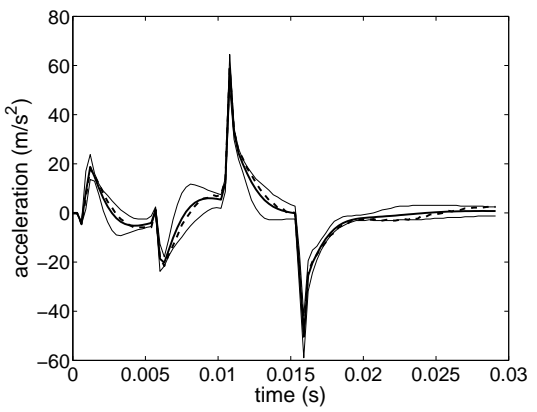

Fig. 13 Random transient acceleration of point $P_{\text {obs }}$, Case 1: confidence region (upper and lower thin solid lines), mean response (thick solid line) and response of the nominal model (dashed line); $x_{0,3}$-acceleration.

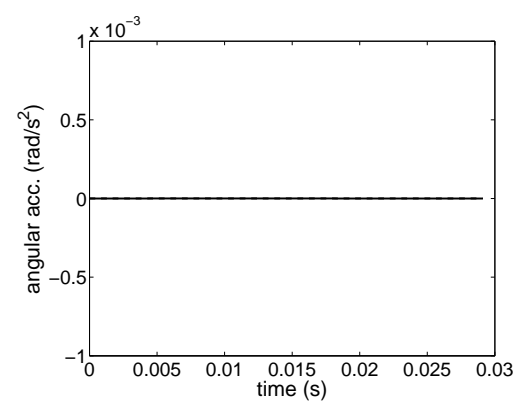

Fig. 14 Random transient acceleration of point $P_{o b s}$, Case 1: confidence region (upper and lower envelopes), mean response and response of the nominal model are superimposed; angular acceleration in $x_{0,1}$-direction.

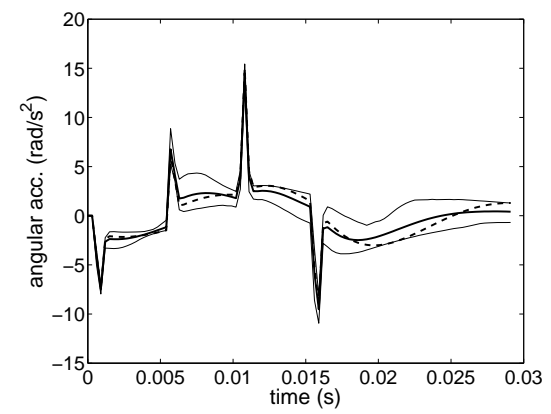

Fig. 15 Random transient acceleration of point $P_{o b s}$, Case 1: confidence region (upper and lower thin solid lines), mean response (thick solid line) and response of the nominal model (dashed line); angular acceleration in $x_{0,2}$-direction. 


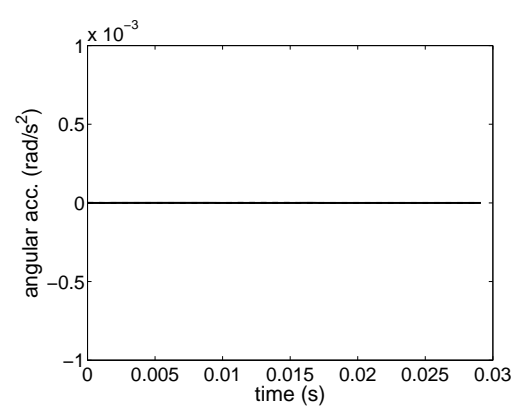

Fig. 16 Random transient acceleration of point $P_{o b s}$, Case 1: confidence region (upper and lower envelopes), mean response and response of the nominal model are superimposed; angular acceleration in $x_{0,3}$-direction.

(ii) Case 2: $M_{i}$ is deterministic, $\mathbf{R}_{0,5}$ is deterministic and [ $\left.\mathbf{J}_{5}\right]$ is random.

We choose $\lambda_{l}=-5$ and $\lambda_{u}=-5$ for random matrix $\left[\mathbf{J}_{5}\right]$. The upper bound for random matrix $\left[\mathbf{Z}_{5}\right]$ is defined by $\left[Z_{5}^{\max }\right]=[[9.61,0,0],[0,1.0,0.0625],[0,0,4.98]]$. Parameters $C_{G_{5}}$ and $[\mu]$ are identified using the methodology explained in Section 3.1.3. We then have $C_{G_{5}}=0.67$ and $[\mu]=[[5.78,0.059,-0.046],[0.059,3.34,0.086],[-0.046,0.086,-0.5]]$ is a symmetric non positive matrix. The probability density function of the trace of random matrix $\left[\mathbf{G}_{5}\right]$ is plotted in Fig. 17. The corresponding dispersion parameter $\delta_{\mathbf{G}_{5}}=\left(\frac{1}{3} E\left\{\left\|\left[\mathbf{G}_{5}\right]-\left[I_{3}\right]\right\|^{2}\right\}\right)^{1 / 2}$ of random matrix $\left[\mathbf{G}_{5}\right]$ is equal to 0.45 . The confidence region, with a probability level $P_{c}=0.90$, of the random acceleration of point $P_{o b s}$ is plotted in Figs. 18-23. We can remark, as it was expected, that all the angular accelerations are sensitive to uncertainties on the tensor of inertia.

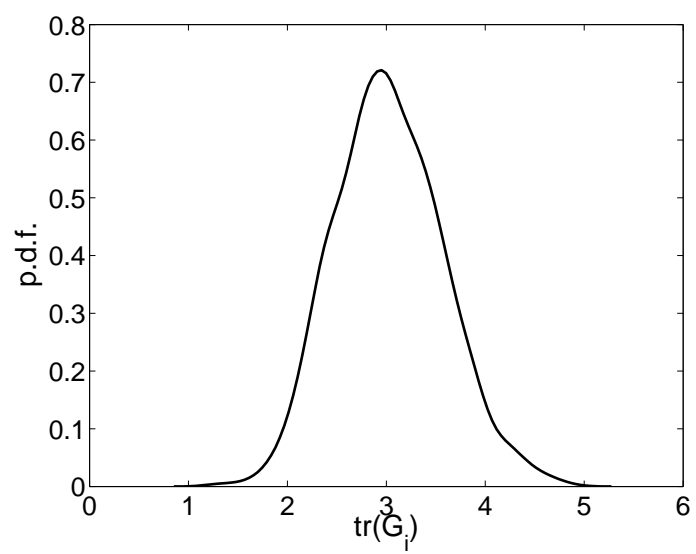

Fig. 17 Probability density function of the trace of random matrix $\left[\mathbf{G}_{5}\right]$. 


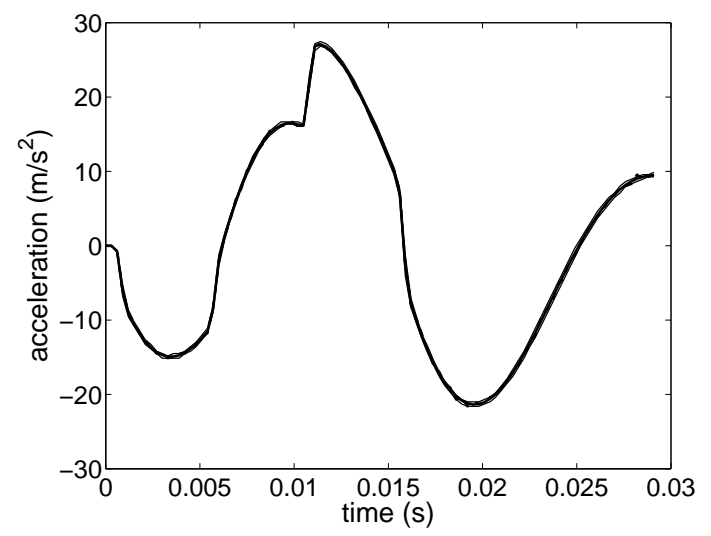

Fig. 18 Random transient acceleration of point $P_{o b s}$, Case 2: confidence region (upper and lower envelopes), mean response and response of the nominal model are superimposed; $x_{0,1^{-}}$ acceleration.

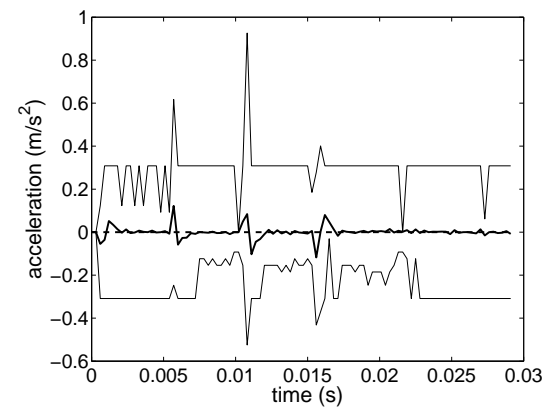

Fig. 19 Random transient acceleration of point $P_{\text {obs }}$, Case 2: confidence region (upper and lower thin solid lines), mean response (thick solid line) and response of the nominal model (dashed line); $x_{0,2}$-acceleration. 


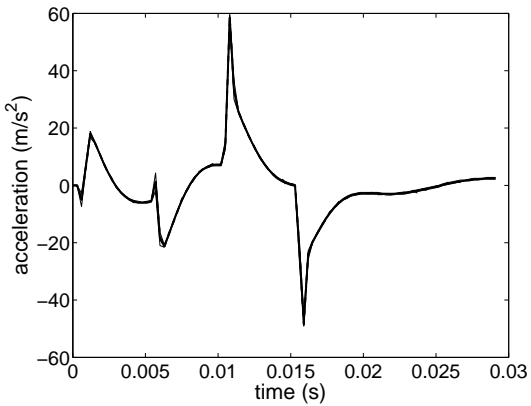

Fig. 20 Random transient acceleration of point $P_{o b s}$, Case 2: confidence region (upper and lower envelopes), mean response and response of the nominal model are superimposed; $x_{0,3^{-}}$ acceleration.

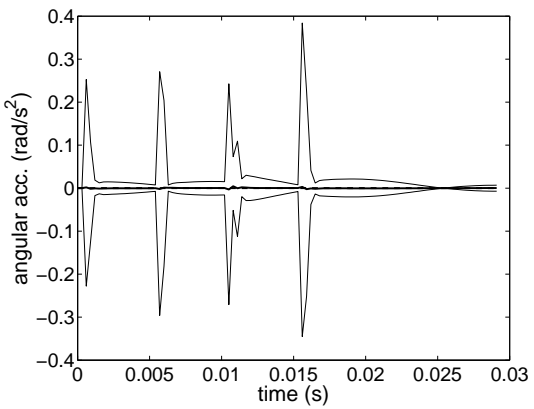

Fig. 21 Random transient acceleration of point $P_{o b s}$, Case 2: confidence region (upper and lower thin solid lines), mean response (thick solid line) and response of the nominal model (dashed line); angular acceleration in $x_{0,1}$-direction. 


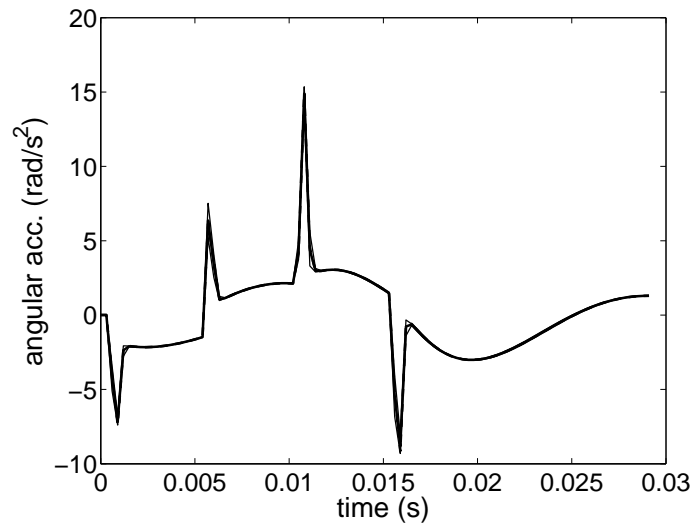

Fig. 22 Random transient acceleration of point $P_{o b s}$, Case 2: confidence region (upper and lower envelopes), mean response and response of the nominal model are superimposed; angular acceleration in $x_{0,2}$-direction.

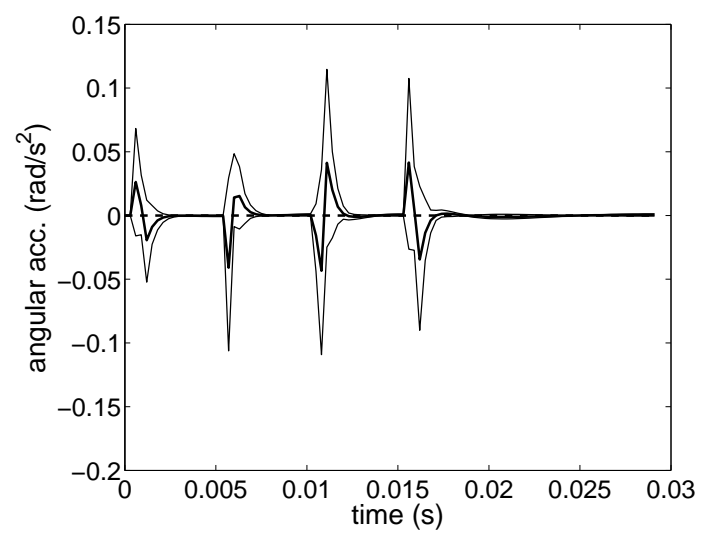

Fig. 23 Random transient acceleration of point $P_{o b s}$, Case 2: confidence region (upper and lower thin solid lines), mean response (thick solid line) and response of the nominal model (dashed line); angular acceleration in $x_{0,3}$-direction.

(iii) Case 3: $M_{5}$ is deterministic, $\mathbf{R}_{0,5}$ is random and $\left[\mathbf{J}_{5}\right]$ is deterministic.

The domain of $\mathbf{R}_{0,5}$ is supposed to be a parallelepiped which is centered at point $(0,0,0.55)$ for which its edges are parallel to the directions $x_{0,1}, x_{0,2}$ and $x_{0,3}$ and for which the lengths following these three directions are respectively $0.5,0.2$ and 0.02. The mean value of random vector $\mathbf{R}_{0,5}$ does not correspond to vector $(0,0,0.55)$. Therefore the PDF of $\mathbf{R}_{0,5}$ is not uniform and depends on the parameters $C_{0}$ and $\lambda_{r}$ which have to be identified solving Eq. (21). We then obtain the values $C_{0}=472$ and $\boldsymbol{\lambda}_{r}=(-2.13,0.77,-7.7)$. Independent realizations of $\mathbf{R}_{0,5}$ are constructed using the Metropolis-Hastings algorithm. Figures 24-26 shows a sampling made up of 500 realizations of the random initial center of mass. We can remark that $\mathbf{R}_{0,5}$ is quasi- 
uniform because the center of the domain of the random initial center of mass $\mathbf{R}_{0,5}$ is near from the mean value $\underline{\mathbf{r}}_{0,5}$.

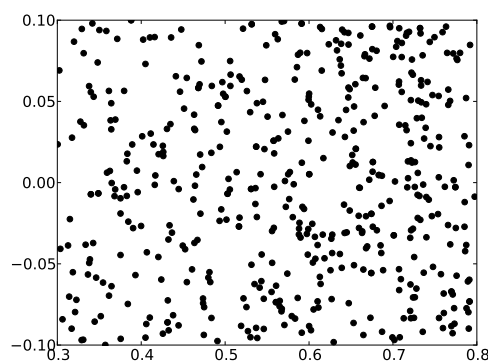

Fig. 24500 realizations of the random initial center of mass: $x_{0,1}-x_{0,2}$ view.

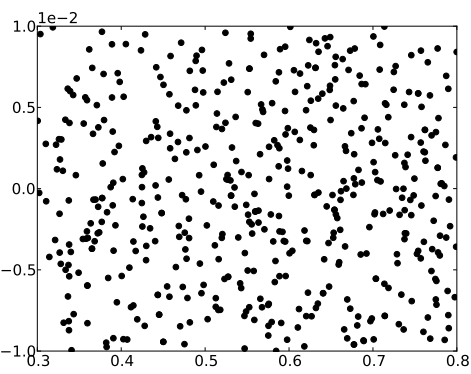

Fig. 25500 realizations of the random initial center of mass: $x_{0,1}-x_{0,3}$ view.

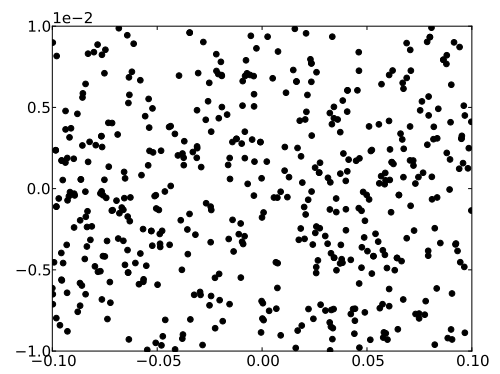

Fig. 26500 realizations of the random initial center of mass: $x_{0,2}-x_{0,3}$ view. 
The confidence region, with a probability level $P_{c}=0.90$, of the random acceleration of point $P_{o b s}$ is plotted in Figs. 27-32. We can remark that all accelerations and all angular accelerations are sensitive to uncertainties on initial center of mass of $R b 5$. The angular accelerations are very sensitive to these uncertainties.

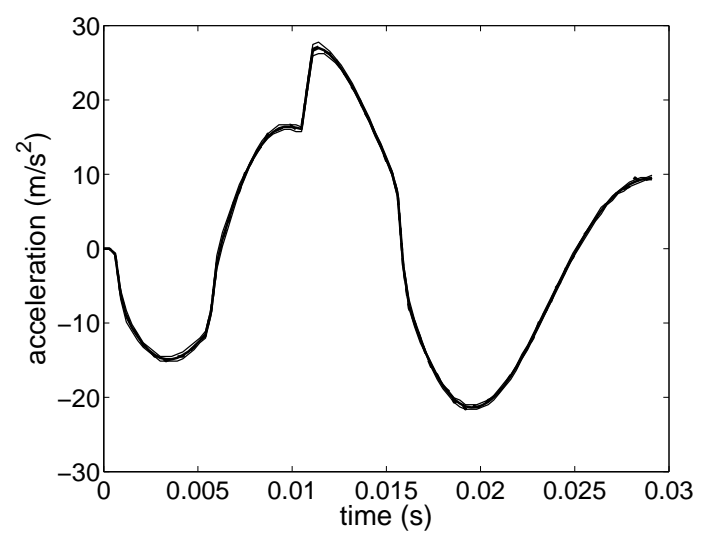

Fig. 27 Random transient acceleration of point $P_{\text {obs }}$, Case 3: confidence region (upper and lower envelopes), mean response and response of the nominal model are superimposed; $x_{0,1^{-}}$ acceleration.

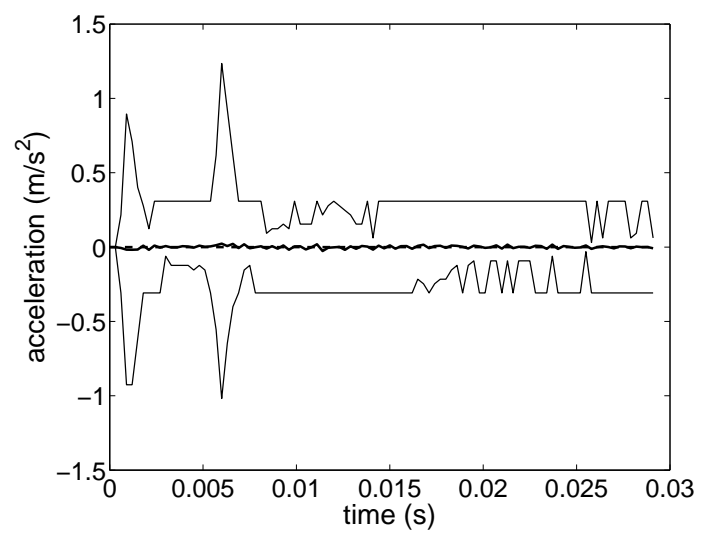

Fig. 28 Random transient acceleration of point $P_{o b s}$, Case 3: confidence region (upper and lower thin solid lines), mean response (thick solid line) and response of the nominal model (dashed line); $x_{0,2}$-acceleration. 


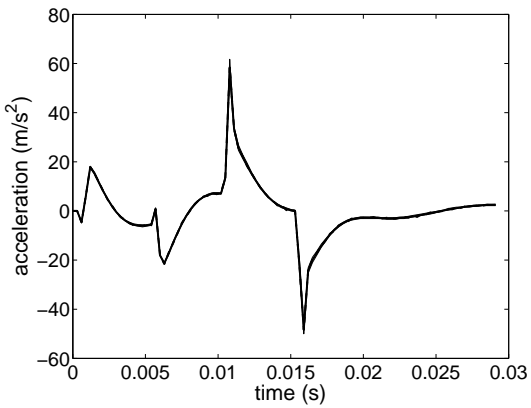

Fig. 29 Random transient acceleration of point $P_{o b s}$, Case 3: confidence region (upper and lower envelopes), mean response and response of the nominal model are superimposed; $x_{0,3^{-}}$ acceleration.

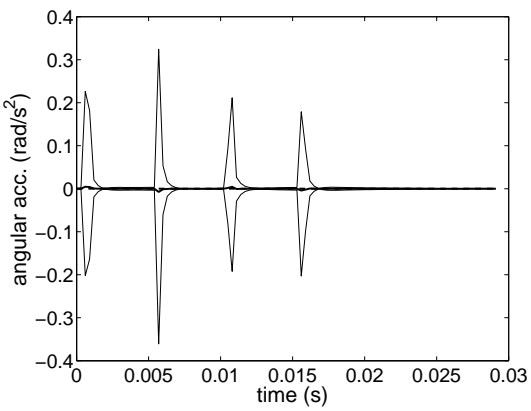

Fig. 30 Random transient acceleration of point $P_{o b s}$, Case 3: confidence region (upper and lower thin solid lines), mean response (thick solid line) and response of the nominal model (dashed line); angular acceleration in $x_{0,1}$-direction.

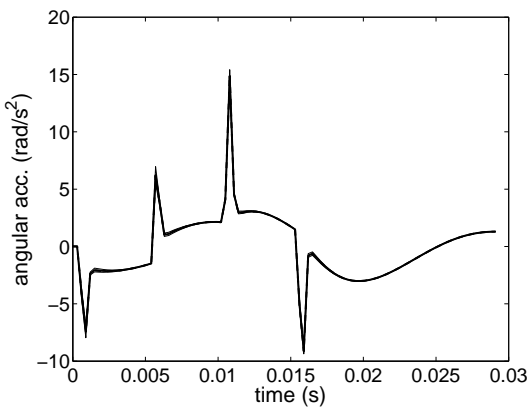

Fig. 31 Random transient acceleration of point $P_{o b s}$, Case 3: confidence region (upper and lower envelopes), mean response and response of the nominal model are superimposed; angular acceleration in $x_{0,2}$-direction. 


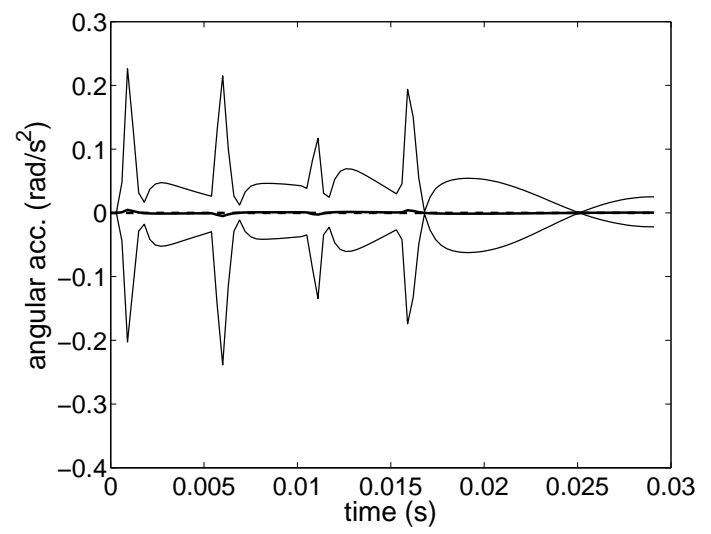

Fig. 32 Random transient acceleration of point $P_{o b s}$, Case 3: confidence region (upper and lower thin solid lines), mean response (thick solid line) and response of the nominal model (dashed line); angular acceleration in $x_{0,3}$-direction.

(iv) Case 4: $M_{5}, \mathbf{R}_{0,5}$ and $\left[\mathbf{J}_{5}\right]$ are random.

The values of the parameters of the PDF are those fixed in the three previous cases. The confidence region, with a probability level $P_{c}=0.90$, of the random acceleration of point $P_{o b s}$ is plotted in Figs. 33-38. It can be viewed that (1) the randomness on the accelerations is mainly due to the randomness of mass $M_{5},(2)$ the randomness on the angular accelerations is mainly due to the randomness of the initial position $\mathbf{R}_{0,5}$ of the center of mass and the random tensor of inertia $\left[\mathbf{J}_{5}\right]$.

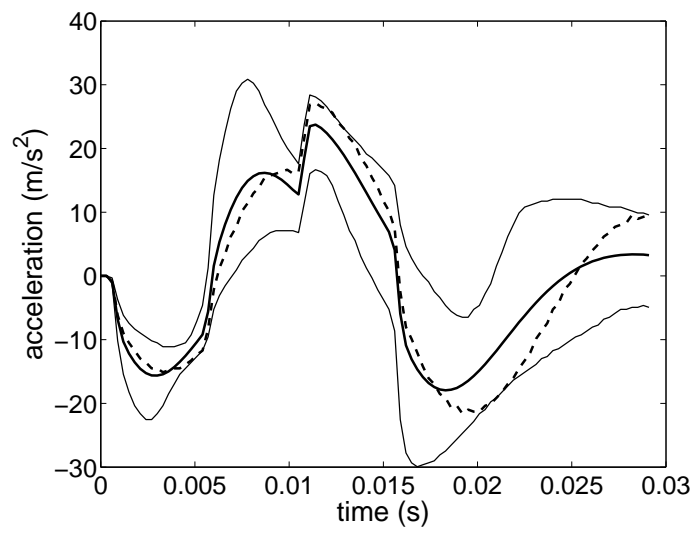

Fig. 33 Random transient acceleration of point $P_{\text {obs }}$, Case 4: confidence region (upper and lower thin solid lines), mean response (thick solid line) and response of the nominal model (dashed line); $x_{0,1}$-acceleration. 


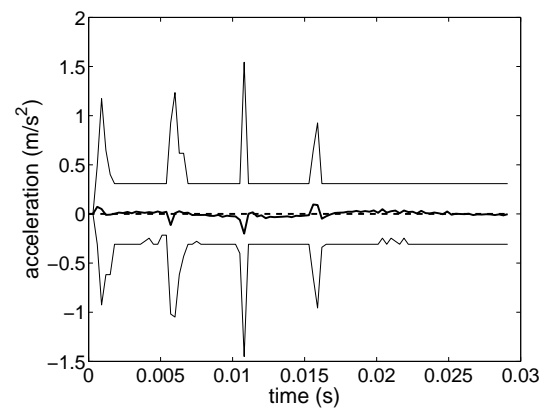

Fig. 34 Random transient acceleration of point $P_{\text {obs }}$, Case 4: confidence region (upper and lower thin solid lines), mean response (thick solid line) and response of the nominal model (dashed line); $x_{0,2}$-acceleration.

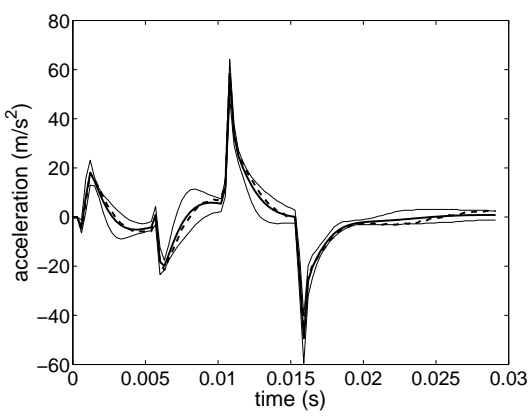

Fig. 35 Random transient acceleration of point $P_{o b s}$, Case 4: confidence region (upper and lower thin solid lines), mean response (thick solid line) and response of the nominal model (dashed line); $x_{0,3}$-acceleration.

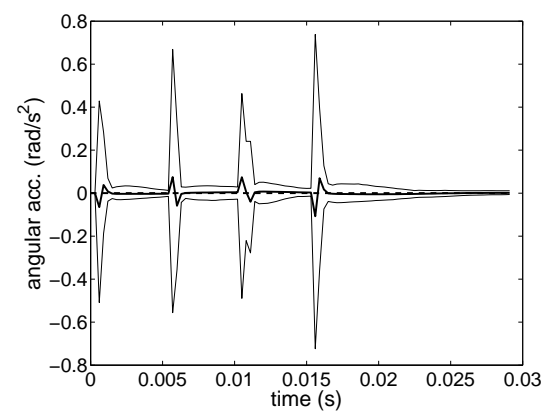

Fig. 36 Random transient acceleration of point $P_{\text {obs }}$, Case 4: confidence region (upper and lower thin solid lines), mean response (thick solid line) and response of the nominal model (dashed line); angular acceleration in $x_{0,1}$-direction. 


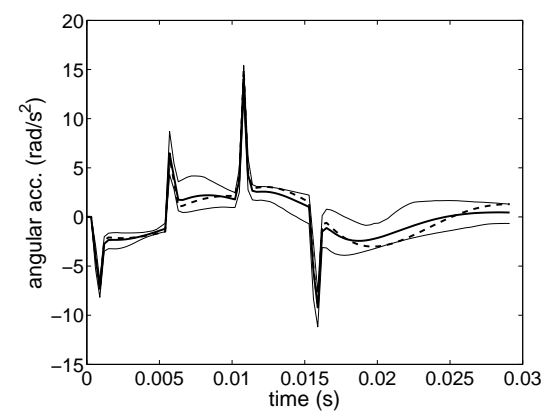

Fig. 37 Random transient acceleration of point $P_{\text {obs }}$, Case 4: confidence region (upper and lower thin solid lines), mean response (thick solid line) and response of the nominal model (dashed line); angular acceleration in $x_{0,2}$-direction.

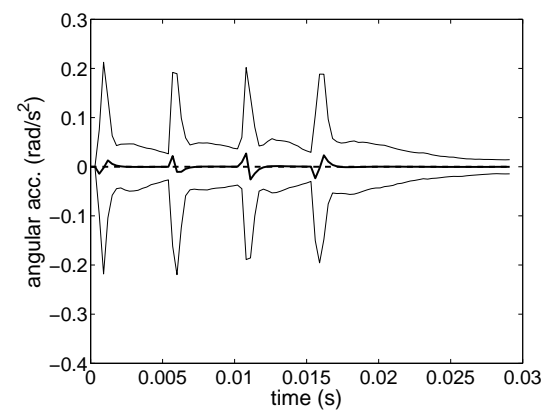

Fig. 38 Random transient acceleration of point $P_{o b s}$, Case 4: confidence region (upper and lower thin solid lines), mean response (thick solid line) and response of the nominal model (dashed line); angular acceleration in $x_{0,3}$-direction.

\section{Conclusion}

We have presented a complete and general probabilistic modeling of uncertain rigid bodies taking into account all the geometrical, mechanical and mathematical properties allowing an uncertain rigid body to be defined in the context of multibody dynamics. This probabilistic model of uncertainties is used to construct the stochastic equations of multibody dynamical systems with uncertain rigid bodies. The random dynamical responses can then be calculated. In the proposed probabilistic model, the mass, the center of mass and the tensor of inertia are modeled by random variables for which the prior probability density functions are constructed using the maximum entropy principle under the constraints defined by all the available mathematical, mechanical and design properties. Several uncertain rigid bodies can be joined to each other in order to obtain the stochastic dynamical model of the uncertain multibody dynamical system. The theory proposed has been illustrated analyzing a simple example. The results obtained clearly show the role played by uncertainties and the sensitivity of the responses due to uncertainties on (1) the mass (2) the center of mass and (3) the 
tensor of inertia. Such a prior stochastic model allows the robustness of the responses to be analyzed with respect to uncertainties. If experimental data were available on the responses, then the parameters which control the level of uncertainties could be estimated by solving an inverse stochastic problem.

\section{Appendix A: Proof of the existence of a physical rigid body corresponding to a given positive definite matrix $[H]$.}

Let $[H]$ be a given positive-definite $(3 \times 3)$ real matrix. Let $[J]$ be the $(3 \times 3)$ real positive definite matrix defined by $[J]=-[H]+\operatorname{tr}([H])\left[I_{3}\right]$. We then have $[H]=-[J]+(\operatorname{tr}([J]) / 2)\left[I_{3}\right]$. Since $[J]$ is positive definite, there exists a matrix $[P]$ such that $[A]=[P]^{T}[J][P]$ is a positivedefinite diagonal matrix. Let $[\widetilde{A}]$ be the positive-definite matrix defined by $[\widetilde{A}]=[P]^{T}[H][P]=$ $-[A]+(\operatorname{tr}([A]) / 2)\left[I_{3}\right]$, we then have $[A]=-[\widetilde{A}]+\operatorname{tr}([\widetilde{A}])\left[I_{3}\right]$. Let $\mathbf{y}=[P] \mathbf{x}$ be the position vector defined in a frame $\left(0, Y_{1}, Y_{2}, Y_{3}\right)$ for which the axes are defined by the columns of matrix $[P]$.

We are looking for a rigid body for which the matrix of its tensor of inertia expressed in the frame $\left(0, Y_{1}, Y_{2}, Y_{3}\right)$ is equal to $[A]$, for which the center of mass is point $(0,0,0)$ and for which the mass is $m_{i}$. To perform such a construction, we introduce a rigid body $\mathrm{RB}_{i}$ made up of 6 material points with equal masses $m_{i} / 6$. The masses are assumed to be localized in the axes. The positions of the 6 masses with respect to frame $\left(0, Y_{1}, Y_{2}, Y_{3}\right)$ are $\left(\ell_{1}, 0,0\right),\left(-\ell_{1}, 0,0\right)$, $\left(0, \ell_{2}, 0\right),\left(0,-\ell_{2}, 0\right),\left(0,0, \ell_{3}\right)$ and $\left(0,0,-\ell_{3}\right)$ where the lengths $\ell_{1}, \ell_{2}$ and $\ell_{3}$ are unknown. It can easily be deduced that the centrum of mass is $(0,0,0)$ and the total mass is $m_{i}$. Then, the matrix $\left[A_{i}\right]$ of the tensor of inertia of rigid body $\mathrm{RB}_{i}$ expressed in frame $\left(0, Y_{1}, Y_{2}, Y_{3}\right)$ is such that

$$
\left[A_{i}\right]=\frac{m_{i}}{6}\left[\begin{array}{ccc}
2 \ell_{2}^{2}+2 \ell_{3}^{2} & 0 & 0 \\
0 & 2 \ell_{3}^{2}+2 \ell_{1}^{2} & 0 \\
0 & 0 & 2 \ell_{1}^{2}+2 \ell_{2}^{2}
\end{array}\right] .
$$

Then, matrix $[A]$ is equal to $\left[A_{i}\right]$ if we have

$$
\begin{aligned}
& \ell_{1}^{2}=3\left(A_{22}+A_{33}-A_{11}\right) /\left(2 m_{i}\right), \\
& \ell_{2}^{2}=3\left(A_{33}+A_{11}-A_{22}\right) /\left(2 m_{i}\right), \\
& \ell_{3}^{2}=3\left(A_{11}+A_{22}-A_{33}\right) /\left(2 m_{i}\right),
\end{aligned}
$$

Therefore, Eq. (54) has a solution if the components of matrix $[A]$ verify the inequalities

$$
\begin{aligned}
& A_{22}+A_{33}-A_{11}>0, \\
& A_{33}+A_{11}-A_{22}>0, \\
& A_{11}+A_{22}-A_{33}>0
\end{aligned}
$$

Since, the diagonal matrix $[\widetilde{A}]$ is positive definite, the conditions defined by Eq. (55) are verified and then Eq. (54) can be solved. Finally, matrix $[J]=[P][A][P]^{T}$ can be interpreted as the matrix of the inertial tensor of rigid body $\mathrm{RB}_{i}$ for which the center of mass is $(0,0,0)$ and for which the mass is $m_{i}$. Consequently, rigid body $\mathrm{RB}_{i}$ exists.

Appendix B: Generator of independent realizations for normalized symmetric positive-definite random matrices.

In this appendix, we recall the formulation [29] for the generator of independent realizations of normalized symmetric positive-definite random matrix $\left[\mathbf{G}_{0, i}\right]$ for which the PDF is defined by 
Eq. (40). The statistical fluctuations of $\left[\mathbf{G}_{0, i}\right]$ is controlled by the dispersion parameter $\delta_{G_{0, i}}$ which is defined by

$$
\delta_{G_{0, i}}=\left\{\frac{1}{3} E\left\{\left\|\left[\mathbf{G}_{0, i}\right]-\left[I_{3}\right]\right\|\right\}_{F}^{2}\right\}^{1 / 2}=\left\{\frac{2}{2+\lambda}\right\}^{1 / 2},
$$

and which must be chosen such that $0<\delta_{G_{0, i}}<\sqrt{1 / 2}$. The probability density function $p_{\left[\mathbf{G}_{0, i}\right]}([G])$ of random matrix $\left[\mathbf{G}_{0, i}\right]$ is rewritten as

$$
p_{\left[\mathbf{G}_{0, i}\right]}([G])=\mathbb{1}_{\mathbb{M}_{3}^{+}(\mathbb{R})}([G]) \times C_{G_{0, i}} \times(\operatorname{det}[G])^{2\left(\delta_{G_{0, i}}^{-2}-1\right)} \times e^{-2 \delta_{G_{0, i}}^{-2} \operatorname{tr}[G]},
$$

in which the positive constant $C_{G_{0, i}}$ is such that

$$
C_{G_{0, i}}=(2 \pi)^{-3 / 2}\left(2 \delta_{G_{0, i}}^{-2}\right)^{6 \delta_{G_{0, i}}^{-2}}\left\{\Pi_{j=1}^{3} \Gamma\left(\frac{2}{\delta_{G_{0, i}}^{2}}+\frac{1-j}{2}\right)\right\}^{-1},
$$

where $\Gamma(z)$ is the gamma function defined for $z>0$ by $\Gamma(z)=\int_{0}^{+\infty} t^{z-1} e^{-t} d t$. Note that Eq. (57) shows that $\left\{\left[\mathbf{G}_{0, i}\right]_{j k}, 1 \leq j \leq k \leq 3\right\}$ are dependent random variables. The random matrix $\left[\mathbf{G}_{0, i}\right]$ is written as $\left[\mathbf{G}_{0, i}\right]=\left[\mathbf{L}_{i}\right]^{T}\left[\mathbf{L}_{i}\right]$, in which $\left[\mathbf{L}_{i}\right]$ is an upper triangular random matrix with values in $\mathbb{M}_{3}(\mathbb{R})$ such that:

(1) the random variables $\left\{\left[\mathbf{L}_{i}\right]_{j j^{\prime}}, j \leq j^{\prime}\right\}$ are independent;

(2) for $j<j^{\prime}$, real-valued random variables $\left[\mathbf{L}_{i}\right]_{j j^{\prime}}$ can be written as $\left[\mathbf{L}_{i}\right]_{j j^{\prime}}=\sigma_{m} U_{j j^{\prime}}$ in which $\sigma_{m}=\delta_{G_{0, i}} / 2$ and where $U_{j j^{\prime}}$ is a real-valued Gaussian random variable with zero mean and variance equal to 1 ;

(3) for $j=j^{\prime}$, positive-valued random variables $\left[\mathbf{L}_{i}\right]_{j j}$ can be written as $\left[\mathbf{L}_{i}\right]_{j j}=\sigma_{m} \sqrt{2 V_{j}}$ in which $V_{j}$ is a positive-valued gamma random variable whose probability density function $p_{V_{j}}(v)$ with respect to $d v$ is written as

$$
p_{V_{j}}(v)=\mathbb{1}_{\mathbb{R}^{+}}(v) \frac{1}{\Gamma\left(\frac{2}{\delta_{G_{0, i}}^{2}}+\frac{1-j}{2}\right)} v^{2 \delta_{G_{0, i}}^{-2}-(1+j) / 2} e^{-v},
$$

in which $\delta_{G_{0, i}}$ is the dispersion parameter defined by Eq. (56).

\section{Appendix C: Properties of random matrix $\left[\mathrm{J}_{i}\right]$ induced by the available information related to random matrix $\left[Z_{i}\right]$.}

In this appendix, we prove that the available information defined by Eq. (35) yields the properties defined by Eq. (44).

(1) From Eqs. (31), (15-a) and (35-a), it can be deduced that random matrix $\left\{\operatorname{tr}\left(\left[\mathbf{J}_{i}\right]\right)\left[I_{3}\right] / 2-\right.$ $\left.\left[\mathbf{J}_{i}\right]\right\}$ is positive definite.

(2) From Eqs. (35-b) and (28), it can be deduced that random matrix $\left(\operatorname{tr}\left(\left[Z_{i}^{\max }\right]\right)\left[I_{3}\right]-\right.$ $\left.\left[Z_{i}^{\max }\right]\right)-\left(\operatorname{tr}\left(\left[\mathbf{Z}_{i}\right]\right)\left[I_{3}\right]-\left[\mathbf{Z}_{i}\right]\right)$ is positive definite. Then, using Eqs. (15-a), (32) and (45) it can be deduced Eq. (44-b)

(3) Since the random variable $M_{i}$ and $\left[\mathbf{Z}_{i}\right]$ are independent, and taking into account Eqs. (15-b) and (35-c), taking the mathematical expectation of Eq. (32), it can be deduced that $E\left\{\left[\mathbf{J}_{i}\right]\right\}=\underline{m}_{i}\left\{\operatorname{tr}\left(\underline{Z}_{i}\right)\left[I_{3}\right]-\left[\underline{Z}_{i}\right]\right\}$. Finally, substituting Eq. (34) in the previous equation yields Eq. (44-c).

(4) Firstly, from Eqs. (36) and (37), it can be deduced that

$$
E\left\{\left\|\left[\mathbf{Z}_{i}\right]^{-1}\right\|_{F}^{2}\right\} \leq C_{L, i} E\left\{\left\|\left[\mathbf{G}_{i}\right]^{-1}\right\|_{F}^{2}\right\},
$$


where $C_{L, i}=\left\|\left[\underline{L}_{Z_{i}}\right]^{-1}\right\|_{F}^{2} \times\left\|\left[\underline{L}_{Z_{i}}\right]^{-T}\right\|_{F}^{2}$. In the PDF defined by Eq. (40), if $\lambda$ is chosen such that $\lambda<-2$, then it can be proven [29] that $E\left\{\left\|\left[\mathbf{G}_{0, i}\right]^{-1}\right\|_{F}^{2}\right\}<+\infty$. Since for $\lambda=\lambda_{l}$ and $[\mu]=\left[\mu_{0}\right]$, the PDF defined by Eqs. (40)-(41) have the same behaviour in the neighborhood of the zero matrix, it can be deduced that $E\left\{\left\|\left[\mathbf{G}_{i}\right]^{-1}\right\|_{F}^{2}\right\}<+\infty$ and then, from Eq. (60),

$$
E\left\{\left\|\left[\mathbf{Z}_{i}\right]^{-1}\right\|_{F}^{2}\right\}<+\infty .
$$

Secondly, it can be shown that if a symmetric real matrix $[A]$ is positive definite with dimension larger than 2, then $\left\|\left(\operatorname{tr}([A])\left[I_{3}\right]-[A]\right)^{-1}\right\|_{F}^{2}<\left\|[A]^{-1}\right\|_{F}^{2}$. Therefore, Eq. (61) yields

$$
E\left\{\left\|\left(\operatorname{tr}\left(\mathbf{Z}_{i}\right)\left[I_{3}\right]-\left[\mathbf{Z}_{i}\right]\right)^{-1}\right\|_{F}^{2}\right\}<+\infty .
$$

Thirdly, since the random variable $M_{i}$ and $\left[\mathbf{Z}_{i}\right]$ are independent and from Eq. (32), it can be deduced that $E\left\{\left\|\left[\mathbf{J}_{i}\right]^{-1}\right\|_{F}^{2}\right\}=E\left\{M_{i}^{-2}\right\} E\left\{\left\|\left(\operatorname{tr}\left(\mathbf{Z}_{i}\right)\left[I_{3}\right]-\left[\mathbf{Z}_{i}\right]\right)^{-1}\right\|_{F}^{2}\right\}$. Then, using subsection 3.1.1-(i) and Eq. (62), the previous equation yields $E\left\{\left\|\left[\mathbf{J}_{i}\right]^{-1}\right\|_{F}^{2}\right\}<+\infty$. Finally, the inequality $E\left\{\left\|\left[\mathbf{J}_{i}\right]^{-1}\right\|^{2}\right\} \leq E\left\{\left\|\left[\mathbf{J}_{i}\right]^{-1}\right\|_{F}^{2}\right\}$ yields Eq. (44-d).

\section{Appendix D: Metropolis-Hastings algorithm.}

Independent realizations of random vector $\mathbf{R}_{0, i}$ and random matrix $\left[\mathbf{G}_{i}\right]$ are obtained using Metropolis-Hasting algorithm . For the readability of the paper, we summarize this algorithm [8]. Let $\mathbf{X}$ be a $\mathbb{R}^{d}$-valued random variable for which the PDF is denoted by $p_{\mathbf{X}}(\mathbf{x})$. Let $q_{\mathbf{X} \mid \mathbf{X}_{k}}\left(\mathbf{x}, \mathbf{x}_{k}\right)$ denote a transition kernel. Let $\mathbf{x}^{(0)}$ and $\mathbf{x}^{(k)}$ denote respectively the initial and the $k^{t h}$ realization of random vector $\mathbf{X}$. Then, the $(k+1)^{t h}$ realization $\mathbf{x}^{(k+1)}$ of random vector $\mathbf{X}$ is generated by the following steps:

(1) Generate a realization $\widetilde{\mathbf{x}}$ of the random vector $\widetilde{\mathbf{X}}$ with the $\operatorname{PDF} \mathbf{x} \mapsto q_{\mathbf{X} \mid \mathbf{x}_{k}}\left(\mathbf{x}, \mathbf{x}^{(k)}\right)$.

(2) Compute $\alpha\left(\mathbf{x}^{(k)}, \widetilde{\mathbf{x}}\right)=\min \left\{1, p_{\mathbf{X}}(\widetilde{\mathbf{x}}) q_{\mathbf{X} \mid \mathbf{X}_{k}}\left(\mathbf{x}^{(k)}, \widetilde{\mathbf{x}}\right) / p_{\mathbf{X}}\left(\mathbf{x}^{(k)}\right) q_{\mathbf{X} \mid \mathbf{X}_{k}}\left(\widetilde{\mathbf{x}}, \mathbf{x}^{(k)}\right)\right\}$.

(3) Generate a realization $z$ of a real-valued random variable $Z$ which is uniform on $[0,1]$.

(4) Test: If $z<\alpha\left(\mathbf{x}^{(k)}, \widetilde{\mathbf{x}}\right)$, then $\mathbf{x}^{(k+1)}=\widetilde{\mathbf{x}}$, else, return to step (1).

A popular choice for the transition kernel is $q_{\mathbf{X} \mid \mathbf{X}_{k}}\left(\mathbf{x}, \mathbf{x}_{k}\right)=g\left(\mathbf{x}-\mathbf{x}_{k}\right)$, where $g$ is a multivariate distribution verifying $g(\mathbf{y})=g(-\mathbf{y})$ for all $\mathbf{y}$ in $\mathbb{R}^{d}$ and thus, $\alpha\left(\mathbf{x}^{(k)}, \widetilde{\mathbf{x}}\right)$ can be rewritten as

$$
\alpha\left(\mathbf{x}^{(k)}, \widetilde{\mathbf{x}}\right)=\min \left\{1, \frac{p_{\mathbf{X}}(\widetilde{\mathbf{x}})}{p_{\mathbf{X}}\left(\mathbf{x}^{(k)}\right)}\right\} .
$$

Then, $\widetilde{\mathbf{X}}$ is a random vector which can be written as $\widetilde{\mathbf{X}}=\mathbf{x}^{(k)}+\mathbf{W}$ in which $\mathbf{W}$ is the centered random variable for which its PDF is $g$. In this case, the algorithm is referred as the Random Walk Metropolis-Hastings algorithm. The distribution $g$, is generally chosen as a centered multivariate Gaussian or as a centered uniform distribution.

\section{References}

1. Batou, A., Soize, C.: Experimental identification of turbulent fluid forces applied to fuel assemblies using an uncertain model and fretting-wear estimation. Mechanical Systems and Signal Processing. 23, 2141-2153 (2009).

2. Baumgarte, J.: Stabilization of constraints and integrals of motion in dynamical systems. Computer Methods in Applied Mechanics and Engineering. 1(1), 1-16 (1972).

3. Carrarini, A.: Reliability based analysis of the crosswind stability of railway vehicles. Journal of Wind Engineering and Industrial Aerodynamics. 95, 493-509 (2007).

4. Chebli, H., Soize, C.: Experimental validation of a nonparametric probabilistic model of non homogeneous uncertainties for dynamical systems. Journal of the Acoustical Society of America. 115(2), 697-705 (2004).

5. Das, S., Ghanem, R.: A bounded random matrix approach for stochastic upscaling. Multiscale Modeling \& Simulation. 8(1), 296-325 (2009) 
6. Chen, C., Duhamel, D., and Soize, C.: Probabilistic approach for model and data uncertainties and its experimental identification in structural dynamics: Case of composite sandwich panels. Journal of Sound and Vibration. 294(12), 64-81 (2006).

7. Durand, J.-F., Soize, C., and Gagliardini, L.: Structural-acoustic modeling of automotive vehicles in presence of uncertainties and experimental identification and validation. J. Acoust. Soc. Am. 124(3), 1513-1525 (2008).

8. Hastings, W.K.: Monte Carlo sampling methods using Markov chains and their applications. Biometrika. 109, 57-97, 1970.

9. Guilleminot, J., Noshadravanb, A., Soize, C., Ghanem, R.: A probabilistic model for bounded elasticity tensor random fields with application to polycrystalline microstrcutures. Computer Methods in Applied Mechanics and Engineering. 200(17-20), 1637-1648, 2011.

10. Haug, E.J.: Computer Aided Kinematics and Dynamics. Vol I: Basic Methods. Allyn and Bacon, Boston (1989).

11. Jaynes, E.T.: Information theory and statistical mechanics. Physical Review. 106(4), 620630 and 108(2), 171-190 (1957).

12. Kaipio, J., Somersalo, E.: Statistical and Computational Inverse Problems. SpringerVerlag, New York (2005).

13. Li, L., Corina Sandu, C.: On the impact of cargo weight, vehicle parameters, and terrain characteristics on the prediction of traction for off-road vehicles. Journal of Terramechanics. 44, 221-238 (2007).

14. Masarati, P., Lanz, M., Mantegazza, P.: Multistep integration of ordinary, stiff and differential-algebraic problems for multibody dynamics applications. $16^{-t h}$ Congresso Nazionale AIDAA. 24-28 Septembre, Palermo, Italy (2001).

15. Murthy, R., Mignolet, M.P., El-Shafei, A.: Nonparametric stochastic modeling of uncertainty in rotordynamics-Part I: Formulation. J. Eng. Gas Turbines Power. 132(9), 092501 (2010).

16. Murthy, R., Mignolet, M.P., El-Shafei, A.: Nonparametric stochastic modeling of uncertainty in rotordynamics-Part II: Applications. J. Eng. Gas Turbines Power. 132(9), 092502 (2010).

17. Nagar, D.K, Gupta, A.K.: Matrix-variate Kummer-Beta distribution. Journal of the Australian Mathematical Society. 73, 11-25 (2002).

18. Negrut, D., Datar, M., Gorsich., D., Lamb, D.: A framework for uncertainty quantification in nonlinear multi-body system dynamics. Proceedings of the 26th Army Science Conference. Orlando, FL (2008).

19. Rubinstein, R.Y.: Simulation and the Monte Carlo Method. John Wiley and Sons (1980).

20. Sandu, A., Sandu, C., Ahmadian, M.: Modeling multibody dynamic systems with uncertainties. Part I: theoretical and computational aspects. Multibody System Dynamics, 23, 375-395 (2006).

21. Sandu, A., Sandu, C., Ahmadian, M., Modeling multibody dynamic systems with uncertainties. Part II: numerical applications. Multibody System Dynamics, 22, 241-262 (2006).

22. Schiehlen, W.: Multibody Systems Handbook. Springer-Verlag, Berlin (1990).

23. Schiehlen, W.: Multibody system dynamics: roots and perspectives. Multibody System Dynamics. 1, 149-188 (1997).

24. Schmitt, K.P., Anitescu, M ., Negrut, D.: Efficient sampling for spatial uncertainty quantification in multibody system dynamics applications. Int. J. Numer. Meth. Engng. 80, 537-564 (2009).

25. Schueller, G.I.: Computational methods in stochastic mechanics and reliability analysis. Computer Methods in Applied Mechanics and Engineering. 194(12-16), 1251-1795 (2005).

26. Serfling, R. J.: Approximation Theorems of Mathematical Statistics. John Wiley \& Sons (1980).

27. Shannon, C.E.: A mathematical theory of communication. Bell System Technology Journal. 27, 379-423 and 623-659 (1948).

28. Soize, C.: A nonparametric model of random uncertainties on reduced matrix model in structural dynamics. Probabilistic Engineering Mechanics. 15(3), 277-294 (2000).

29. Soize, C.: Maximum entropy approach for modeling random uncertainties in transient elastodynamics. J. Acoust. Soc. Am. 109(5), 1979-1996 (2001).

30. Soize, C.: Random matrix theory for modeling random uncertainties in computational mechanics. Comput. Methods Appl. Mech. Eng. 194(1216), 1333-1366 (2005).

31. Soize, C.: Construction of probability distributions in high dimension using the maximum entropy principle. Applications to stochastic processes, random fields and random matrices. International Journal for Numerical Methods in Engineering. 76(10), 1583-1611 (2008). 
32. Soize, C., Capiez-Lernout, E., Durand, J.F., Fernandez, C., Gagliardini, L.: Probabilistic model identification of uncertainties in computational models for dynamical systems and experimental validation. Computer Methods in Applied Mechanics and Engineering. 198(1), 150-163 (2008). 